\title{
Investigation of a polychromatic tomography method for the extraction of the three-dimensional spatial structure of implosion core plasmas
}

Cite as: Phys. Plasmas 19, 082705 (2012); https://doi.org/10.1063/1.4743017

Submitted: 13 June 2012 . Accepted: 24 July 2012 . Published Online: 08 August 2012

T. Nagayama, R. C. Mancini, R. Florido, D. Mayes, R. Tommasini, J. A. Koch, J. A. Delettrez, S. P. Regan, and V. A. Smalyuk

\section{ARTICLES YOU MAY BE INTERESTED IN}

Processing of spectrally resolved $x$-ray images of inertial confinement fusion implosion cores recorded with multimonochromatic $x$-ray imagers

Journal of Applied Physics 109, 093303 (2011); https://doi.org/10.1063/1.3580513

Understanding reliability and some limitations of the images and spectra reconstructed from a multi-monochromatic $x$-ray imager

Review of Scientific Instruments 86, 113505 (2015); https://doi.org/10.1063/1.4935828

Multiobjective method for fitting pinhole image intensity profiles of implosion cores driven by a Pareto genetic algorithm

Review of Scientific Instruments 77, 10F525 (2006); https://doi.org/10.1063/1.2338314

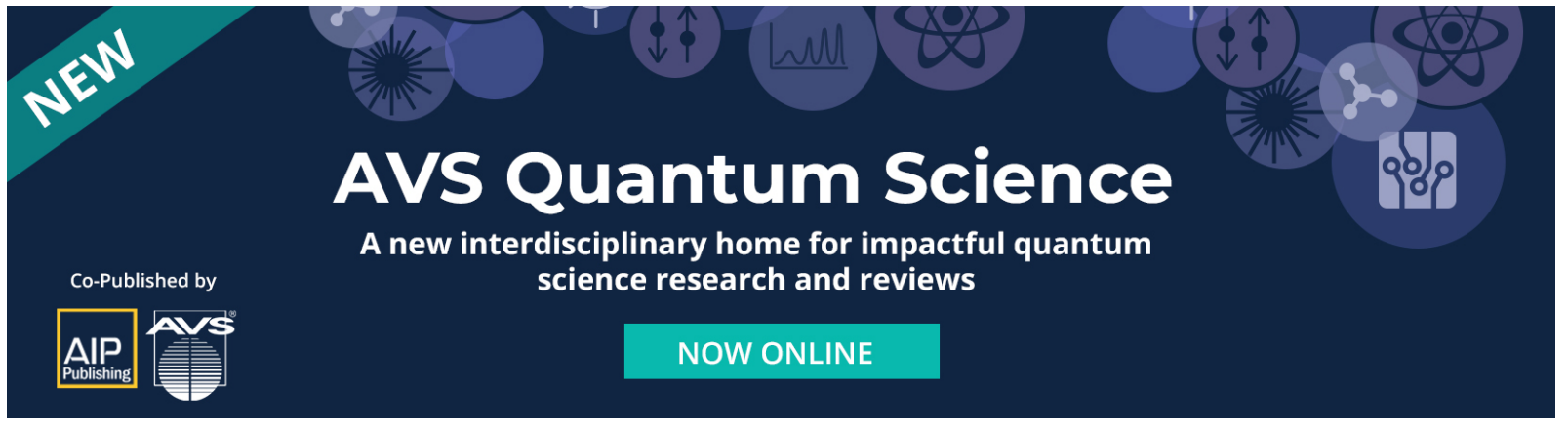




\title{
Investigation of a polychromatic tomography method for the extraction of the three-dimensional spatial structure of implosion core plasmas
}

\author{
T. Nagayama, ${ }^{1, a)}$ R. C. Mancini, ${ }^{1}$ R. Florido, ${ }^{1, b)}$ D. Mayes, ${ }^{1}$ R. Tommasini, ${ }^{2}$ J. A. Koch, ${ }^{2}$ \\ J. A. Delettrez, ${ }^{3}$ S. P. Regan, ${ }^{3}$ and V. A. Smalyuk ${ }^{2,3}$ \\ ${ }^{1}$ Physics Department, University of Nevada, Reno, Nevada 89557, USA \\ ${ }^{2}$ Lawrence Livermore National Laboratory, Livermore, California 94550, USA \\ ${ }^{3}$ Laboratory for Laser Energetics, University of Rochester, New York 14623, USA
}

(Received 13 June 2012; accepted 24 July 2012; published online 8 August 2012)

\begin{abstract}
A spectroscopic method has been developed to extract the three-dimentional spatial structure (i.e., spatial distribution of electron temperature and density) of inertial confinement fusion implosion cores based on the analysis of space-resolved spectra from a tracer element recorded along three quasi-orthogonal lines of sight. We discuss a spectral model that computes space-resolved spectra for a given spatial structure. This model is then combined with a multi-objective search and optimization technique driven by a Pareto genetic algorithm to perform the inversion and to extract the spatial structure of the implosion core from a simultaneous and self-consistent analysis of a set of space-resolved spectra. This method is investigated with a series of synthetic data test cases to explore its reliability, requirements, and limitations. We have found a constraint parameter $\mathrm{P}_{\text {const }}$ such that the method is robust and the extracted spatial structure is reliable when $P_{\text {const }}>1$. The idea of polychromatic tomography is general and has potential to extract the spatial structure of other laboratory high energy-density plasmas. (C) 2012 American Institute of Physics. [http://dx.doi.org/10.1063/1.4743017]
\end{abstract}

\section{INTRODUCTION}

Inertial confinement fusion (ICF) is an approach to compress a millimeter-size spherical shell capsule containing fuel (e.g., deuterium, tritium) to temperature and density conditions suitable for ignition. ${ }^{1-4}$ The capsule is symmetrically heated directly or indirectly by laser beams, and as the capsule surface ablates, the rest of it is compressed as a reaction to the ablation. At stagnation, the implosion kinetic energy is turned into fuel thermal energy and a central hot spot is formed. When appropriate temperature and density spatial distributions are achieved, thermonuclear fusion starts in the hot spot and a burning front propagates outwards to heat and burn the colder and denser fuel surrounding the hot spot. For the last few decades, significant progress has been made in theory, experiments, and diagnostics in high energy density physics in an effort to better understand the physics relevant to ICF and to achieve ignition.

To design ICF experiments, the implosion dynamics has to be studied using hydrodynamics simulations, which are complex models taking into account multidisciplinary physics in extreme conditions. Thus, diagnostics are crucial to benchmark hydrodynamics models and to connect theory and experiment for a better understanding of ICF physics. In past several decades, $x$-ray spectroscopy has proved to be a powerful tool to extract average electron temperature and density of implosion core plasmas. ${ }^{5-10}$ However, to better understand experiments and benchmark hydrodynamics

\footnotetext{
${ }^{a)}$ Present address: Sandia National Laboratories, Albuquerque, New Mexico 87185-1193, USA.

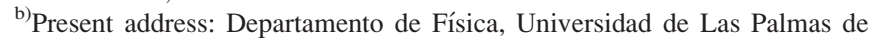
Gran Canaria, 35017 Las Palmas de Gran Canaria, Spain.
}

models, average temperature and density diagnostics are not sufficient. It is important to diagnose if the experiments achieved the temperature and density spatial distributions as designed, or if not, to understand why and to find out how different they are. This demand is increasing as ICF research advances. For example, in addition to conventional centralhot-spot ignition, two alternate ignition approaches are under investigation, namely fast ignition ${ }^{11,12}$ and shock ignition. ${ }^{11,13,14}$ All these approaches require different spatial structures, ${ }^{15}$ and there is no general method to measure them.

The goal of this paper is to investigate a spectroscopic method to extract the three-dimensional spatial structure of ICF implosion cores based on the simultaneous analysis of three sets of space-resolved spectra recorded along quasiorthogonal lines of sight, and to explore the reliability, requirements, and limitations of the method. Our approach relies on line emissions from a tracer element (e.g., argon), which is added to the fuel for diagnostic purposes. Section II describes argon-doped ICF experiments and discusses the challenges found in previous one- and two-dimensional spatial structure analysis. Section III describes the threedimensional spatial structure analysis method that successfully overcame the challenges discussed in Sec. II by simultaneously and self-consistently analyzing sets of spaceresolved spectra recorded along three quasi-orthogonal lines of sight. First, we discuss the calculation of emissivity and opacity of the argon tracer for the relevant experimental conditions (Sec. III A), and then we introduce a geometrically adaptable radiation transport model (RADIATOR) that computes sets of synthetic space-resolved spectra based on given temperature and density distributions defined in an arbitrary 
volume (Sec. III B). This model is combined with a powerful multi-objective inversion algorithm called PGALM (Sec. III C), which consists of a forward reconstruction search and optimization driven by a Pareto genetic algorithm (PGA) ${ }^{16,17}$ followed up by a Levenberg-Marquardt non-linear leastsquares minimization method (LM). ${ }^{18}$ The method is first tested with a simple case to investigate if the sets of spaceresolved spectra can constrain the three-dimensional spatial structure (Sec. IV). Then, in Sec. V, real data effects were taken into account to perform realistic synthetic data test cases, and the method is further tested by a series of sensitivity studies to find out the method's requirements and limitations. Finally, Sec. VI summarizes our findings and their implications.

\section{ARGON DOPED ICF IMPLOSION EXPERIMENTS AND CORE CONDITION ANALYSIS}

In argon-doped ICF implosion experiments performed at the OMEGA laser facility of the Laboratory for Laser Energetics (LLE) at the University of Rochester, argon K-shell line emission spectroscopy had been applied to diagnose electron temperature and density of the implosion core under various different symmetry assumptions. In those experiments, 60 OMEGA laser beams (a total energy of $\approx 18-23 \mathrm{~kJ})$ were used to irradiate the surface of spherical plastic shell targets $(\mathrm{R} \approx 400 \mu \mathrm{m}$ and $\Delta \mathrm{R} \approx 20-30 \mu \mathrm{m})$ filled with $20 \mathrm{~atm}$ of deuterium. At maximum compression, the implosion core electron temperature is in the range of $500-3000 \mathrm{eV}$, and the electron density in the range of $1 \times 10^{23}-5 \times 10^{24} \mathrm{~cm}^{-3}$. Under these conditions, deuterium is fully ionized and emits continuum radiation that is not very informative to infer temperature and density in the plasma. Thus, a tracer amount of argon (0.1-0.2\% atomic concentration) is added to the target for diagnostic purposes. The amount of argon has to be chosen carefully. It has to be small, so that it does not significantly affect the hydrodynamics of the implosion due to radiation cooling, but it also has to be large enough to produce enough spectroscopic signature for diagnostics. ${ }^{19}$ For these temperature and density conditions, argon is highly ionized, but not fully ionized. The dominant abundances are Li-, He-, and H-like argon ions, and their $\mathrm{K}$-shell emission lines are sensitive to the plasma environmental conditions, i.e., electron temperature and density. The density sensitivity comes from the Stark broadening of the spectral line shapes. The temperature sensitivity comes from the fact that the line intensity ratio of similar line transitions in adjacent ionization stages reflects the ionization balance of the source plasma. For example, argon $\mathrm{He} \beta$ line is due to the transition of a bound electron from $\mathrm{n}=3$ to $\mathrm{n}=1$ from He-like argon, while Ly $\beta$ is the same type of transition in H-like argon. In order to properly model the line intensity spectral distribution, one also has to take into account the contributions from nearby $n=4$ to $n=1$, $\mathrm{n}=5$ to $\mathrm{n}=1$ as well as satellite transitions. Hence, the spectrum computed by a detailed spectral model will show changes in line broadening and relative intensity depending upon temperature and density. Taking advantage of this property, one can uniquely infer the electron temperature and density of the implosion core by fitting synthetic spectra to the measured spectra. ${ }^{5-10}$ Another important consideration is the effect of line radiation transport. For the tracer amount of argon we use, the mean-free-path of $\mathrm{He} \beta$ and $\mathrm{Ly} \beta$ photons are larger than implosion core size (i.e., optical depths $<1$ ), and thus the emergent line intensity distribution have contributions from regions deep inside the core.

In the past, a space-averaged temperature and density of the core had been studied based on the analysis of spaceintegrated argon K-shell line emission spectra. ${ }^{7-10,20,21}$ Recently, the analyses were extended to one- and twodimensions with symmetry assumptions. To this end, argon $\mathrm{He} \beta$ monochromatic images, which are images integrated over the width of the line, were analyzed simultaneously with the space-integrated spectrum. A series of multi-objective data analyses of the line images and space-integrated spectrum successfully extracted electron temperature and density spatial distributions for spherical symmetry, ${ }^{22-24}$ as well as for the case of a collection of core slices with local axial symmetry. ${ }^{25-27}$

The data analyzed by Golovkin $e t a l .{ }^{22}$ were recorded at Osaka University Institute of Laser Engineering using two instruments: streaked $\mathrm{x}$-ray spectrometer to record the spaceintegrated spectrum and $x$-ray monochromatic framing camera to record line intensity images. The data analyzed by Welser et al. ${ }^{25-27}$ and Nagayama et al. ${ }^{23,24,28}$ were recorded at OMEGA with a single instrument called multimonochromatic X-ray imager (MMI). MMI is an instrument that consists of a pinhole array, a multi-layered Bragg mirror, and a gated $\mathrm{x}$-ray framing camera detector. It records a collection of implosion core images each of which is formed by photons in a slightly different energy range. By processing MMI data, one can extract both monochromatic line images and space-integrated spectrum recorded along the same line of sight. ${ }^{29-33}$

Here, we must note two challenges found in previous spatial structure analyses. First, as long as the data are recorded along one line of sight, the analysis has to employ some type of symmetry assumption. Even though pinhole image data have two-dimensional spatial resolution, the data are still integrated along the line of sight. Hence, the more asymmetric the implosion core becomes the less reliable these analyses are. Second, there is a disadvantage in the use of monochromatic line images to diagnose density distribution. Ratios of monochromatic images based on similar line transition from adjacent ionization stages (e.g., $\operatorname{argon} \mathrm{He} \beta$ and $\operatorname{Ly} \beta$ images) provide information on temperature distribution. ${ }^{28}$ However, the electron density sensitivity of the Stark-broadened line shapes is partially lost in images that are integrated over line widths. Thus, the use of monochromatic images is not best suited to extract density spatial distribution.

To overcome these challenges, there were recently major improvements in MMI data recording and processing. First, three identical MMI instruments were fielded along quasi-orthogonal lines-of-sight (LOS) to observe ICF implosion cores simultaneously from different views. ${ }^{34}$ Fig. 1 shows the angular coordinates $(\theta, \phi)$ of the three LOS in the spherical coordinate system. The angles between these 


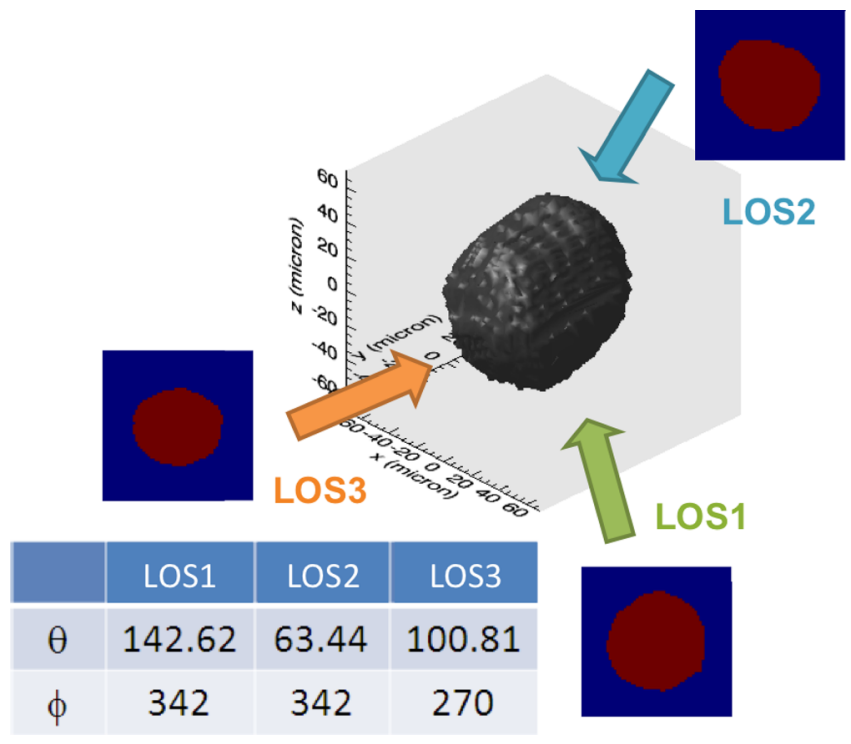

FIG. 1. The three lines of sight (LOS1, LOS2, and LOS3) and their coordinates in the OMEGA chamber.

diagnostic ports are $70.5^{\circ}$ between LOS1 and LOS3, 79.2 between LOS1 and LOS2, and $79.2^{\circ}$ between LOS2 and LOS3. Second, the MMI data processing software was improved. ${ }^{33}$ Not only the quality of the reconstructed images and space-integrated spectrum were improved but also it became possible to extract space-resolved spectra integrated along chords of finite cross-section determined by spatial resolution and signal-to-noise ratio. These chords are illustrated by long rectangular volumes in Fig. 2. One advantage of the use of space-resolved spectra over monochromatic line images is that the spectrum carries both electron temperature and density sensitivity through the line intensities and the Stark broadening of the line shape. Thus, each spaceresolved spectrum has the information of chord-averaged electron temperature and density.

We note that, since the data were collected along three different LOS simultaneously, temperature and density at each volume element (i.e., the intersection of the three long rectangular volumes in Fig. 2) is constrained by an unique combination of three space-resolved spectra recorded along

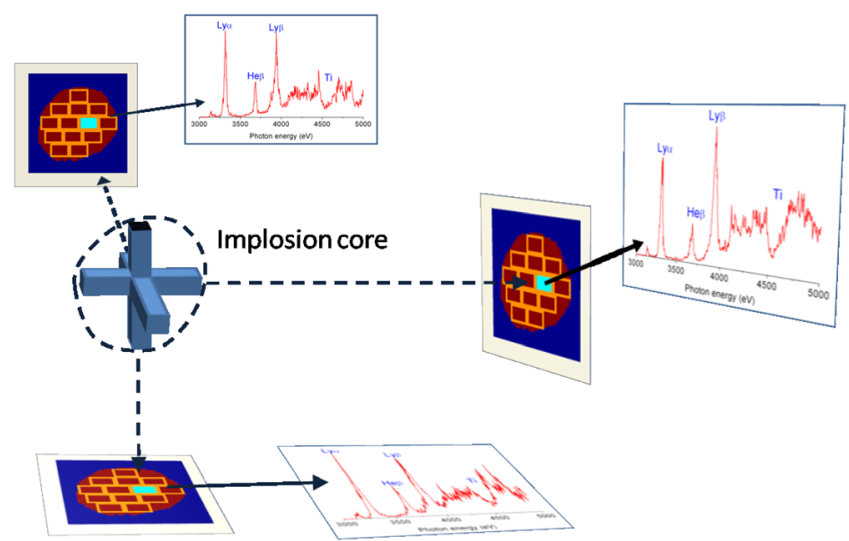

FIG. 2. Each space-resolved spectrum has temperature and density information integrated along chords parallel to the LOS and perpendicular to the image plane. Each spatial region (i.e., volume element) is located at a unique intersection of three chords. Thus, spatial regions are constrained by their contributions to spatially resolved spectra recorded along three LOS. quasi-orthogonal LOS. Thus, all the spatial volume elements are intertwined by their contribution to three sets of space-resolved spectra, and it is important to analyze all these spectra simultaneously and self-consistently to extract the three-dimensional distribution of temperature and density in the implosion core without making symmetry assumptions.

\section{POLYCHROMATIC TOMOGRAPHY METHOD}

\section{A. Calculation of emissivity and opacity}

In order to compute the space-resolved spectra, the emissivity and opacity of the tracer element must be determined. The atomic kinetics calculations for argon ions in ICF implosion core plasmas have been performed with the collisional-radiative atomic kinetics model ABAKO. ${ }^{21,35}$ To solve atomic kinetics of argon ions for this application, ABAKO considered all ionization states from C-like argon to the fully stripped ion and includes up to 4592 energy levels, with the following maximum number of energy levels per ion: 1 fully stripped, $100 \mathrm{H}$-like, $352 \mathrm{He}$-like, $519 \mathrm{Li}$ like, 644 Be-like, 1299 B-like, and 1677 C-like Ar. Energy levels and spontaneous radiative decay rates for line transitions were computed using the atomic structure code FAC (Ref. 36) including unresolved transition arrays ${ }^{37}$ and configuration interaction corrections. The calculations took into account all non-autoionizing and autoionizing states characterized by principal quantum numbers consistent with the continuum lowering criterion. ${ }^{38}$ The radiation transport effect on the atomic kinetics is approximated by the escape factor for spherical geometry using a source radius estimated from a mass conservation argument. ${ }^{39}$ Line shapes include the effects of natural, Doppler, and Stark broadening. Especially, Stark broadening is very important, since it is the main broadening mechanism and relevant for electron density diagnostics. ${ }^{40-43}$ The Stark broadening model used here took into account the perturbation effects on line transitions of both argon and deuterium ions and electrons. Once the level populations have been obtained from the solution of the non-local thermodynamic equilibrium atomic kinetics model, the local radiative properties are computed assuming isotropy, and the values of the emissivity and opacity are tabulated as a function of electron temperature and density. We note that here we work under the approximation of using a set of pre-computed temperature-, density-, and photonenergy-dependent emissivity and opacity tables to model the radiative properties of the plasma source. This approximation is sufficient for the sake of demonstrating the spectroscopic method discussed in this paper.

\section{B. Geometry adaptable radiation transport model}

Once the radiation properties of argon are calculated, the emergent space-resolved spectra can be computed by a geometry adaptable RADIATOR. To compute spaceresolved spectra, RADIATOR requires the following information: temperature and density distributions continuously defined in an arbitrary volume, information of the line of sight, and the spatial regions of interest (for space-resolved spectra), which are defined within the implosion core 


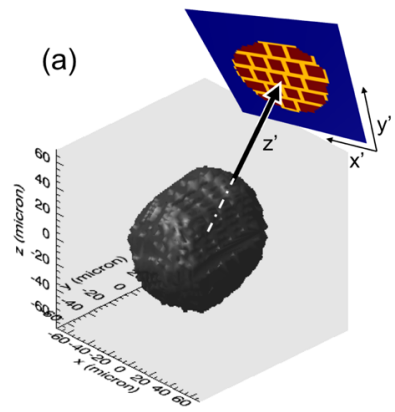

(b)

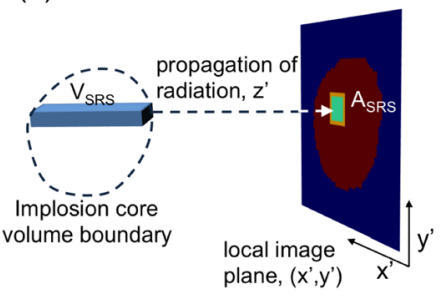

FIG. 3. (a) A continuous temperature and density distributions in an arbitrary shaped implosion core are defined in a global coordinate system $(x, y, z)$, and an arbitrary line of sight and the detector plane determines its local coordinate system $\left(x^{\prime}, y^{\prime}, z^{\prime}\right)$. Spatial regions on the image plane are defined within the boundary of the implosion core projection on to the image plane. (b) One spatial region on the image plane $\left(\mathrm{A}_{\mathrm{SRS}}\right)$ and the corresponding partial volume $\mathrm{V}_{\mathrm{SRS}}$ responsible for its space-resolved spectrum are illustrated in the local coordinate system $\left(x^{\prime}, y^{\prime}, z^{\prime}\right)$. Direction of radiation propagation is defined as $z^{\prime}$, and the local image plane (detector plane) of the line of sight is defined as $x^{\prime}$ and $y^{\prime}$.

projection on the detector plane (Fig. 3(a)). RADIATOR employs cartesian coordinate system, and the shape of the implosion core and the distributions of electron temperature and density could be as flexible and detailed as the computation time permits. Figure 3(a) shows a schematic figure to illustrate an implosion core defined in the target chamber (i.e., global frame of reference) and an arbitrary line of sight. Figure 3(b) shows a LOS specific local frame of reference, which illustrates one of the spatial region of interest, $\mathrm{A}_{\mathrm{SRS}}$, where the subscript stands for space-resolved spectra, and the corresponding partial volume, $\mathrm{V}_{\mathrm{SRS}}$, which is responsible for the emergent space-resolved spectrum assuming parallel ray trace. In the local frame of reference, the direction of the radiation transport is defined as local $z^{\prime}$ axis, and the detector plane, which is perpendicular to the $z^{\prime}$-axis, defines the $x^{\prime}$ and $y^{\prime}$-axes.

Assuming parallel rays, the emergent intensity of photons with frequency $\nu$ at $\left(x^{\prime}, y^{\prime}\right)$ of the detector plane can be computed by numerically integrating the radiation transport equation

$$
\frac{d I_{\nu}\left(x^{\prime}, y^{\prime}\right)}{d z^{\prime}}=\epsilon_{\nu}\left(x^{\prime}, y^{\prime}, z^{\prime}\right)-I_{\nu}\left(x^{\prime}, y^{\prime}\right) \kappa_{\nu}\left(x^{\prime}, y^{\prime}, z^{\prime}\right),
$$

where emissivity, $\epsilon_{\nu}$, and opacity, $\kappa_{\nu}$, at point $\left(x^{\prime}, y^{\prime}, z^{\prime}\right)$ can be retrieved from the emissivity and opacity database computed by ABAKO collisional radiative model as follows:

$$
\begin{aligned}
\epsilon_{\nu}\left(x^{\prime}, y^{\prime}, z^{\prime}\right) & =\epsilon_{\nu}^{A B A K O}\left(T_{e}\left(x^{\prime}, y^{\prime}, z^{\prime}\right), N_{e}\left(x^{\prime}, y^{\prime}, z^{\prime}\right)\right), \\
\kappa_{\nu}\left(x^{\prime}, y^{\prime}, z^{\prime}\right) & =\kappa_{\nu}^{A B A K O}\left(T_{e}\left(x^{\prime}, y^{\prime}, z^{\prime}\right), N_{e}\left(x^{\prime}, y^{\prime}, z^{\prime}\right)\right) .
\end{aligned}
$$

The values of temperature and density at local point $\left(x^{\prime}, y^{\prime}, z^{\prime}\right)$ can be extracted by using the linear transformation discussed by Nagayama et al. $^{33}$ and referencing temperature and density at the corresponding point $(x, y, z)$ in the global frame of reference where the temperature and density distributions are defined (Fig. 3(a)). The emergent space-resolved spectrum associated with a spatial region $\mathrm{A}_{\mathrm{SRS}}$ can be computed by integrating $I_{\nu}$ over the area as follows:

$$
I_{\nu}^{S R S}=\iint_{A_{S R S}} I_{\nu}\left(x^{\prime}, y^{\prime}\right) d x^{\prime} d y^{\prime},
$$

which results in the computation of radiation transport within the rectangular volume indicated in Fig. 3(b). The resultant spectra are further convolved with an instrumental broadening function to take into account the finite spectral resolution of the instrument. The same procedure is repeated for each spatial region $\left(\mathrm{A}_{\mathrm{SRS}}\right)$ defined on each detector plane (for LOS1, LOS2, and LOS3) to compute all of the spaceresolved spectra from given temperature and density distributions.

This model is flexible in the shape of spatial regions $\left(\mathrm{A}_{\mathrm{SRS}}\right)$ defined on the detector planes and can compute emergent space-resolved spectra even for arbitrary-shaped spatial area. The number of extracted space-resolved spectra depends on the discretization of the image planes. Figure 4 shows examples of discretizations on each image plane (i.e., LOS1, LOS2, and LOS3). The difference in the shape of the implosion core projection boundary among LOS1, LOS2, and LOS3 comes from the arbitrariness of the implosion core shape. Also, these projection images can be discretized

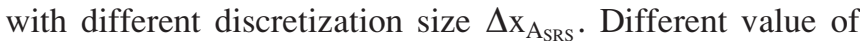
$\Delta \mathrm{x}_{\mathrm{ASRS}}$ results in different number of space-resolved spectra extracted by the data processing and those computed by RADIATOR in the analysis. As $\Delta \mathrm{x}_{\mathrm{A}_{\mathrm{SRS}}}$ becomes smaller, the number of space-resolved spectra increases and provides more spatial information. However, in the case of real data analysis, there are two limitations in the selection of $\Delta \mathrm{x}_{\mathrm{A}_{\mathrm{SRS}}}$.

\section{(a) $\Delta x=21 \mu \mathrm{m} \rightarrow 38$ SRS}

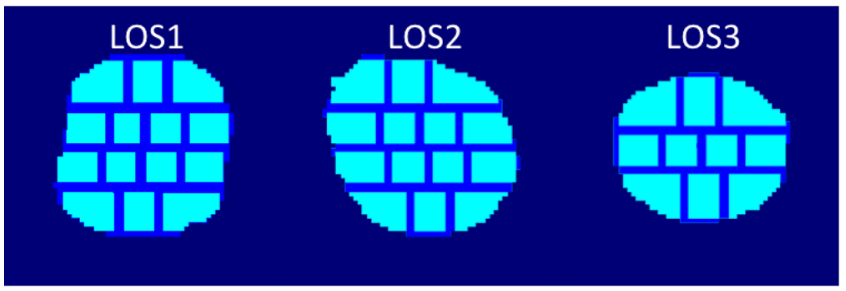

(b) $\Delta x=16 \mu \mathrm{m} \rightarrow 65 \mathrm{SRS}$

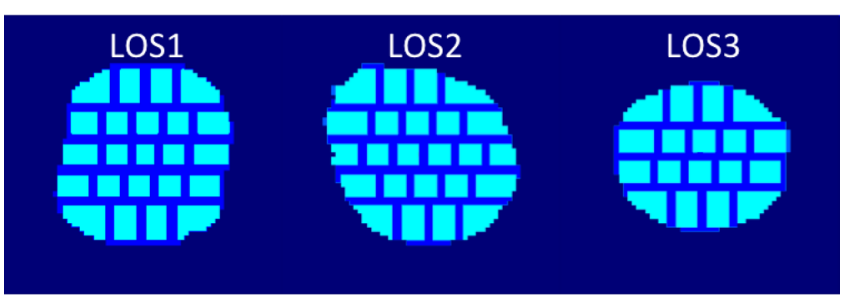

(c) $\Delta \mathrm{x}=11 \mu \mathrm{m} \rightarrow 142 \mathrm{SRS}$

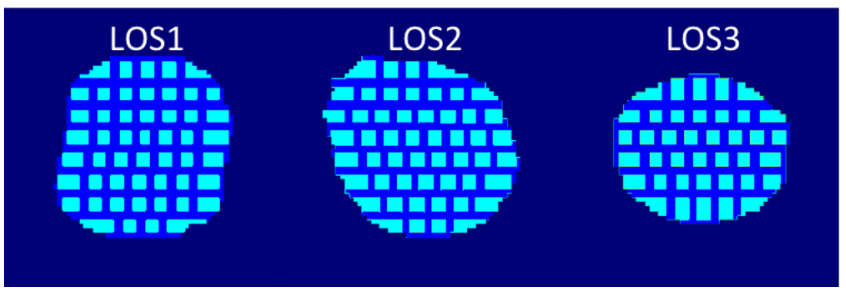

FIG. 4. Examples of discretization on each image plane. (a) $\Delta \mathrm{x}_{\mathrm{A}_{\mathrm{SRS}}}=21 \mu \mathrm{m}$, (b) $\Delta \mathrm{x}_{\mathrm{A}_{\mathrm{SRS}}}=16 \mu \mathrm{m}$, and (c) $\Delta \mathrm{x}_{\mathrm{A}_{\mathrm{SRS}}}=11 \mu \mathrm{m}$. The total number of space-resolve spectra are 38,65 , and 142, respectively. 
One is that, as the spatial region $\mathrm{A}_{\mathrm{SRS}}$ gets smaller, the signal to noise ratio of the extracted space-resolved spectra decreases, so that for a $\Delta \mathrm{x}_{\mathrm{A}_{\mathrm{SRS}}}$ value smaller than a certain threshold, the spectral information becomes unreliable. The other limitation is the finite spatial resolution of the instrument, which is about $10 \mu \mathrm{m}$. Thus, the discretization procedure on the image plane affects the analysis results. This point will be addressed in Sec. V A.

\section{Multi-objective inversion}

The calculation performed by RADIATOR is combined with an efficient multi-objective inversion algorithm to invert the problem and to find the three-dimensional temperature and density distributions from given sets of space-resolved spectra. To this end, an inversion algorithm, which we call PGALM, is used. PGALM starts with a Pareto genetic algorithm, ${ }^{16}$ and the set of solutions are further refined by the Levenberg-Marquardt non-linear least-squares (LM). ${ }^{18}$ Genetic algorithm (GA) is a search and optimization algorithm inspired by the natural selection of evolutionary biology. GA efficiently finds a solution by exploring only a fraction of the search parameter space. Also, since GA begins a search using a set of randomly initialized parameters, there is no bias in the search, and the uniqueness of the solution can be tested by repeating the same search starting with a different initial random seed. Moreover, the PGA - which has been successfully applied to several spectroscopic analyses_- ${ }^{17,22-24,26-28}$ combines a GA with the Pareto optimization scheme for multi-objective optimization. PGA is a fast and robust search algorithm to find good solutions without any bias, however, it is not an efficient technique to find fully converged solutions. Thus, a set of reasonably good solutions found by PGA are further optimized by LM minimization algorithm to quickly achieve final convergence. The combination of PGA and LM complement each other and results in a very efficient search and optimization algorithm. ${ }^{23,24,28}$

Now, in order to solve the inversion problem, the object space (i.e., the implosion core volume) needs to be discretized and this is an important technical aspect to be discussed. RADIATOR requires temperature and density distributions continuously defined in an arbitrarily shaped implosion core, which is critical to minimize the possible numerical discrepancies due to the linear transformation from the global frame to a local frame for an arbitrary line of sight. However, the number of search parameters is limited due to two factors. First, we cannot expect PGALM to find the temperature and density distributions with better resolution than the spatial resolution of the instrument. Second, the time required by PGALM increases roughly proportional to the square of the number of searched parameters $\left(t_{\text {analysis }} \propto \mathrm{N}_{\text {param }}^{2}\right)$. That means 10 times better spatial resolution in the analysis result requires $10^{3}$ times more parameters in the same volume, which would take about $10^{6}$ times longer. Even though PGALM is an efficient inversion algorithm, a good parameterization and a good decoding from parameters into continuous temperature and density distributions are crucial.

For this purpose, the object space is discretized into voxels (i.e., finite size volume element), and a finite number of temperatures and densities are defined at the centers of these voxels as the search parameters. There are two discretization parameters in order to define this discretized implosion core: voxel size $\Delta \mathrm{x}_{\text {voxel }}$ and threshold fraction $f_{t h}$. Each voxel has a volume of $\Delta x_{\text {voxel }}^{3}$. Since the actual implosion core is defined in a smooth and arbitrary shape, which cannot be perfectly represented by voxels, some voxels only partially lie within the actual implosion core volume. Thus, for a given voxel, let denote $f$ as the fraction of its volume inside the implosion core volume. All those voxels with $f>f_{\text {th }}$, being $f_{\text {th }}$ the socalled threshold fraction, will be included as part of the discretized implosion core, and, therefore, their corresponding temperature and density values will be searched. Figure 5 shows how implosion core is discretized depending on different values of $\Delta \mathrm{x}_{\text {voxel }}$ and $f_{t h}$. As $\Delta \mathrm{x}_{\text {voxel }}$ becomes smaller, the number of voxels increases. As a result, the spatial structure can be more flexible, but at the same time, it is harder for the model to constrain the number of temperatures and densities based on the given sets of space-resolved spectra. Also, as the threshold fraction $f_{\text {th }}$ decreases, the spatial structure becomes more flexible at the edge of the core volume. However, voxels with too small fraction should not be included for parameter search, since the space-resolved spectra are not very sensitive to the temperature and density values in such small fraction of the volume. The local conditions in these small volumes can be estimated by extrapolation from its neighbors. The analysis sensitivity to this object space discretization is studied in Sec. V A.

The number of search parameters is the number of the voxels, i.e., $\mathrm{N}_{\text {voxel }}$, multiplied by two (electron temperature and density). The PGALM searches for this reduced number of electron temperatures and densities. Then, the continuous temperature and density distributions are computed based on these temperatures and densities at the center of voxels with tri-linear interpolation.

\section{PROOF-OF-PRINCIPLE CASE}

This section presents a proof-of-principle test case of polychromatic tomography based on synthetic data analysis.
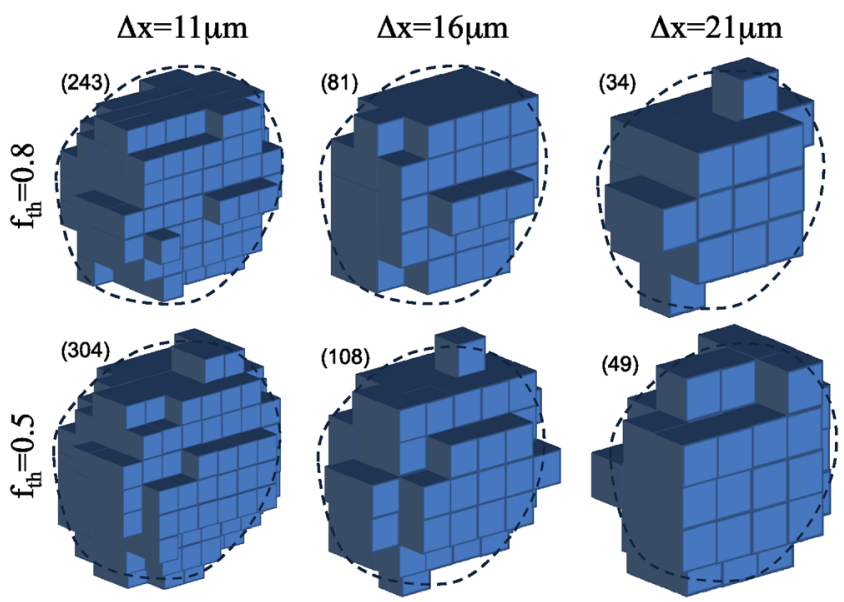

FIG. 5. Discretization of the object space with $\Delta \mathrm{x}_{\mathrm{voxel}}=11,16$, and $21 \mu \mathrm{m}$ and $f_{t h}=0.5$ and 0.8 . The number in the parentheses indicates the number of voxels for each combination of discretization parameters. 
To this end, details associated with the experimental set up and measurements such as quasi-orthogonality in the actual lines of sight, noise in the data, and approximations in the object space discretization and decoding scheme are not taken into account.

The synthetic data are based on a random distribution of temperature and density, which are defined in a $3 \times 3 \times 3$ cube (Fig. 6). The voxel size length is $\mathrm{L}=25 \mu \mathrm{m}$, thus the plasma size is $75 \mu \mathrm{m}$, which is comparable to implosion core sizes in OMEGA experiments. The three LOS are taken to be orthogonal (i.e., $x$-, $y$-, and $z$ - axes) parallel to the sides of the cube, and the three sets of space-resolved spectra are computed along these LOS. Figure 6(a) shows a schematic illustration of the target plasma, the LOS, and the sets of space-resolved spectra. The computed argon spectral range is $3500-4100 \mathrm{eV}$, which covers $\operatorname{argon} \mathrm{He} \beta, \mathrm{He} \gamma$, and $\mathrm{Ly} \beta$ lines. These lines have optical depth less than 1 for the size, temperature, and density ranges of this example. Thus, the emergent line intensity distributions carry information from all depths in the plasma.

Since each line of sight has $3 \times 3=9$ spatial regions of area $A_{S R S}$, a total of 27 space-resolved spectra are computed. At this point, a separate analysis of each spectrum would yield effective or chord-averaged electron temperature and density of the corresponding sub-volume of the plasma, which is defined by the chord or the line of integration with the associated cross-sectional area $\mathrm{A}_{\mathrm{SRS}}$. Thus, by analyzing 27 space-resolved spectra on a separate basis, one could extract 9 effective (or chord-averaged) temperatures and densities along 9 parallel chords on each line of sight. However, this procedure cannot be used to unfold the line integration to determine local temperatures and densities in the voxels.

Using the same data, the test case described in this section shows a novel idea to unfold the line integration and extract local conditions by analyzing 27 space-resolved spectra simultaneously and self-consistently. The PGALM searched for the optimal 27 local temperatures and densities in the voxels such that RADIATOR simultaneously produces the best fits to all of the given space-resolved spectra. Fig. 7 shows the target electron temperature and density distributions, and the analysis result obtained by PGALM. The solution found by PGALM is identical to the target distribution. Furthermore, in order to check the uniqueness of the solution, the analysis of the same synthetic data was repeated several times, each time initializing PGALM with a different random seed. In all cases, PGALM found the same solution.

There are two reasons why this method is able to extract three dimensional temperature and density distributions with the synthetic data associated only with three lines of sight. First, we have multiple parallel chords along each line of sight; for the example shown, we have 9 for each. Second, each chord has information associated with multiple wavelengths, since the data is spectrally resolved. This additional information is important because, even though the information is line integrated, every point along the chord has different emissivity and opacity for different wavelengths. This proof-of-principle case shows that the limited number of lines of sight in this method is compensated by multiple wavelengths. We call this spectroscopic method polychromatic tomography because it takes into account the information encoded in photon frequency (i.e., wavelength or "color") along multiple LOS.

\section{MODEL SENSITIVITY TESTS}

In this section, we will refine the synthetic data test cases and include additional concerns and technical complexities that have to be taken into account for real data analysis. First, in actual experiments, it is often difficult to record the data along perfectly orthogonal lines of sight. Second, temperatures and densities are not defined in the discrete $3 \times$ $3 \times 3$ voxels, but they are continuously changing within an arbitrary shape. This point is challenging because the spatial structure has to be characterized by a finite number of parameters due to the limitations in the spatial resolution of the instruments and the computational time. Third, the actual data have noise, and its potential impact on the method results must be investigated.

In order to address these considerations, the target temperature and density were redefined in more realistic way. The implosion core volume used for these test cases is shown in Fig. 1. Within this volume, continuous target electron temperature and density distributions are defined using skewed three dimensional Gaussian functions as follows:

$$
\begin{aligned}
\mathrm{T}_{\mathrm{e}}[\mathrm{eV}]= & 1900 \times \exp \left\{-\frac{1}{40^{2}}\left(x^{2}+\frac{(y+5)^{2}}{0.8^{2}}+\frac{(z+10)^{2}}{1.2^{2}}\right)\right\} \\
& +500,
\end{aligned}
$$

(a)

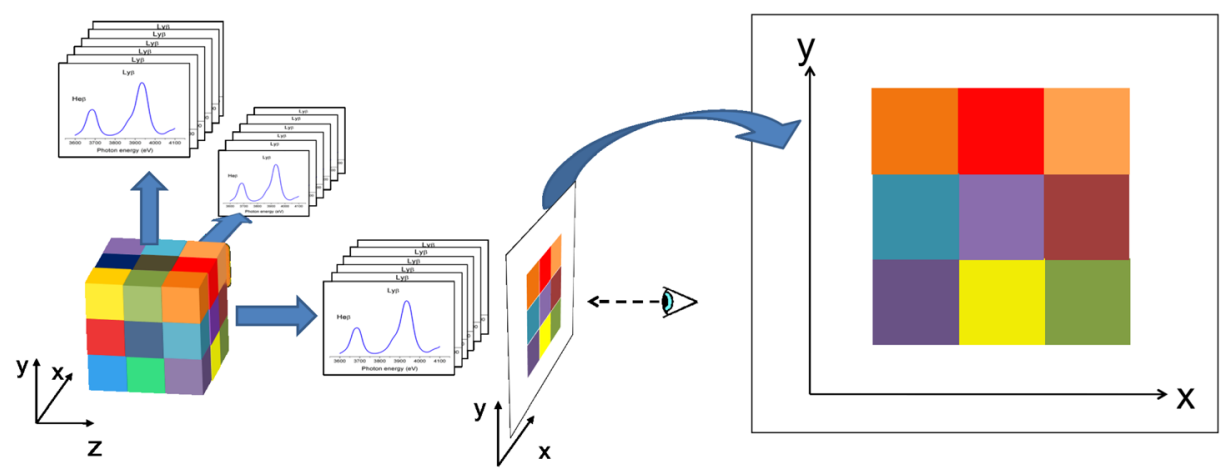

FIG. 6. (a) $3 \times 3 \times 3$ cubic target and the sets of space-resolved spectra, (b) definition of each spatial region on the image plane $\left(\mathrm{A}_{\mathrm{SRS}}\right)$ perpendicular to the $z$-axis and view of the implosion core along the line of sight defined in (a). 
(a) Electron temperature, $\mathrm{T}_{\mathrm{e}}[\mathrm{eV}]$
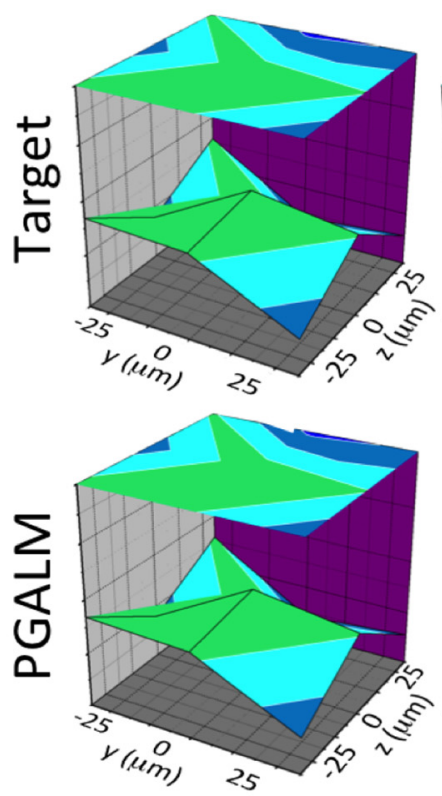

$x=-25 \mu m$
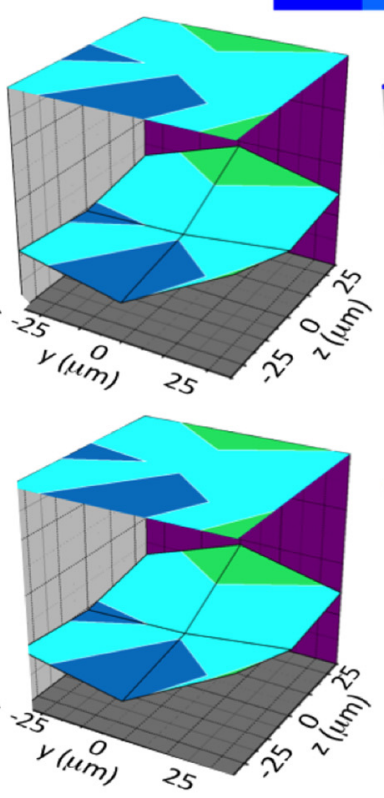

$\mathrm{x}=0 \mu \mathrm{m}$
1000 1500
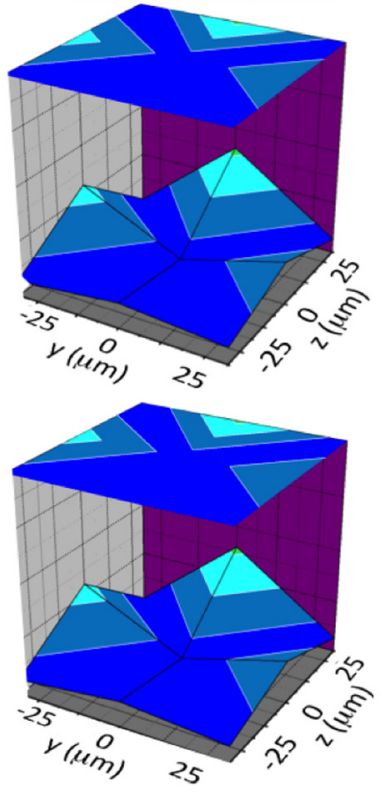

$x=25 \mu \mathrm{m}$

(b) Electron density, $\mathrm{N}_{\mathrm{e}}\left[10^{24} \mathrm{~cm}^{-3}\right]$

$0.001 .25 \quad 2.503 .75 \quad 5.00 \quad 6.25$
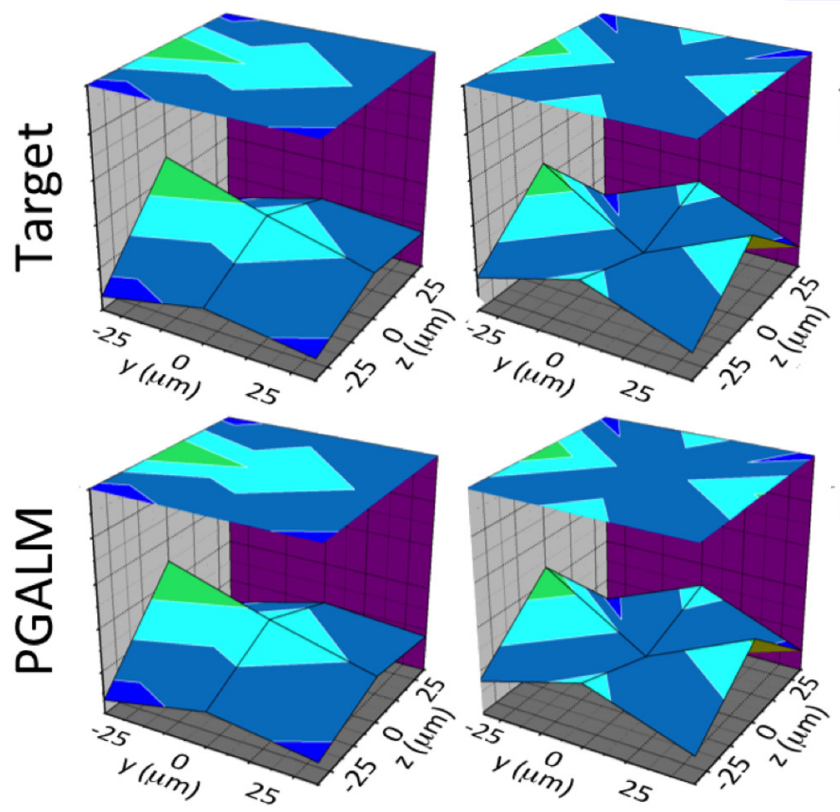

$x=-25 \mu m$

$\mathrm{x}=0 \mathrm{um}$

$\mathrm{N}_{\mathrm{e}}\left[\mathrm{cm}^{-3}\right]=-3.0 \times 10^{24}$

$$
\begin{aligned}
& \times \exp \left\{-\frac{1}{40^{2}}\left(x^{2}+\frac{(y-10)^{2}}{1.2^{2}}+\frac{(z-5)^{2}}{0.8^{2}}\right)\right\} \\
& +4.0 \times 10^{24}
\end{aligned}
$$

where $x, y$, and $z$ are the global coordinates (in $\mu \mathrm{m}$ ) defined in the target chamber. In an effort to be general, the temperature and densitiy are skewed in different directions and have
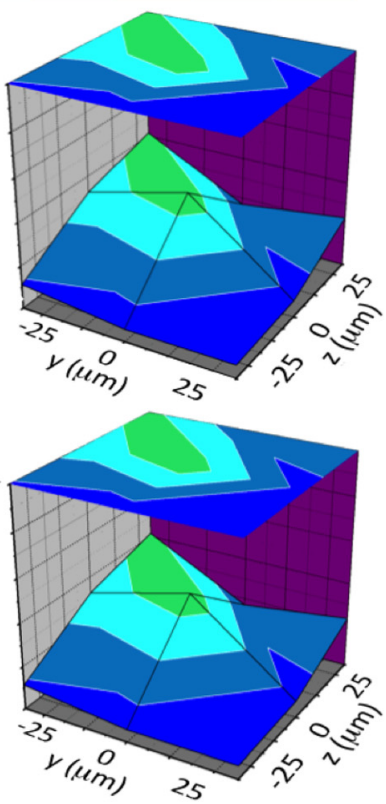

$$
x=25 \mu \mathrm{m}
$$

slightly different centers. The temperature is defined to range between 500 and $2400 \mathrm{eV}$ with its maximum at $(0,-5,-10)$ microns, and the distribution is elongated along the $z$-axis. The density is defined to range between $1.0 \times 10^{24}$ and $3.0 \times 10^{24} \mathrm{~cm}^{-3}$ with its minimum at $(0,10,5)$ microns, and the distribution is elongated along the $y$-axis. Figure 8 shows a tomographic picture of the target electron temperature and density distributions. These asymmetries are created on purpose to check if the PGALM can find these arbitrary 


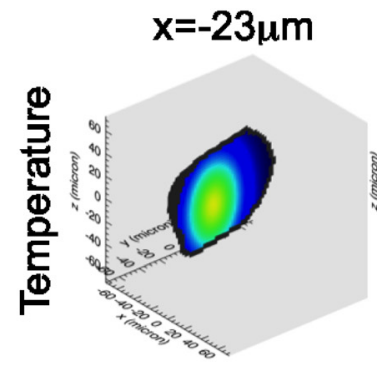

$[\mathrm{eV}]$

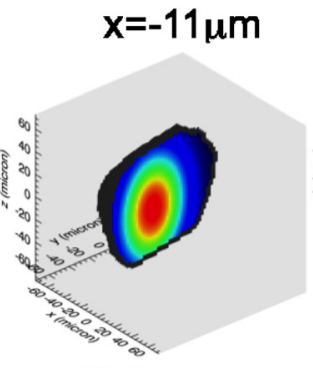

1000

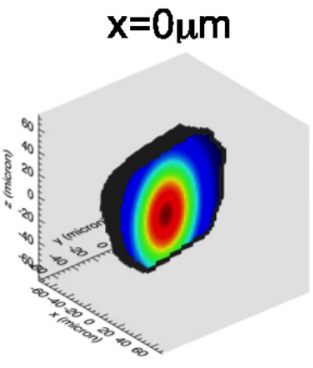

1500

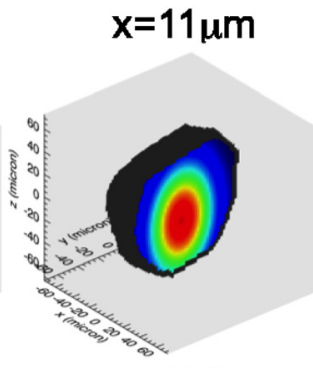

2000

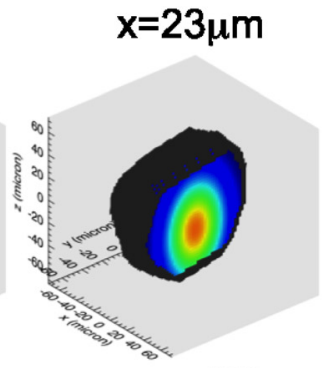

2500
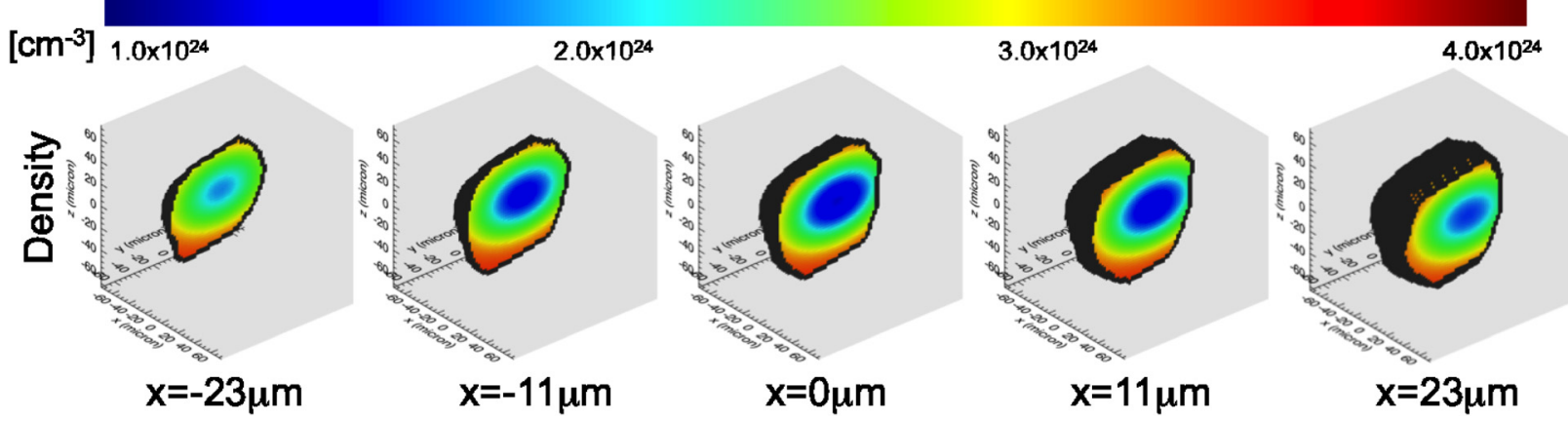

FIG. 8. Cross-sections of synthetic electron temperature and density distributions.

distributions. Also, these analytic functions allow the target distributions to have smooth curvature, which cannot be perfectly reproduced by decoding the limited number of parameters using the tri-linear interpolation discussed in Sec. III C. Thus, these target temperature and density distributions provide a good example to check the robustness and limitations of the parametrization and decoding techniques. RADIATOR employs the actual three lines of sight defined in Fig. 1 to compute the three sets of space-resolved spectra for the given temperature and density distributions (Eqs. (3) and (4)). To be realistic, these target spectra are convolved with the instrumental broadening function to take into account the finite spectral resolution of the MMI instruments.

There are two more factors that could affect these refined synthetic test cases. One is the choice of discretizations: on image plane (Fig. 4) and in object space (Fig. 5). The other is the noise level on the data. Thus, in this section, the method's sensitivity to these two factors is further investigated. These studies are critical to understand the limitations and requirements of the method and to interpret the analysis results properly.

\section{A. Sensitivity to discretizations}

The discretization on the image planes discussed in Sec. III B depends on minimum areal size $\Delta \mathrm{x}_{\mathrm{A}_{\mathrm{SRS}}}$, which determines the size, shape, and locations of all the spatial regions as well as the number of space-resolved spectra. The discretization in the object space (the implosion core) discussed in Sec. IIIC depends on voxel size $\Delta \mathrm{x}_{\mathrm{voxel}}\left(\right.$ or $\Delta \mathrm{x}_{\mathrm{vxl}}$ ) and threshold fraction $f_{t h}$. The combination of these parameters defines the number of voxels and how many temperatures and densities are searched for. The success of the analysis depends on the choice of these discretizations. The spatial structure of the target implosion core is studied with different combinations of discretizations to find out the best discretizations on the image plane and in the object space. For each discretization case, all the space-resolved spectra are computed based on the target temperature and density distributions using RADIATOR. Also, a minimum of 5\% Poisson noise was added.

For the image plane discretization, we tested $\Delta \mathrm{x}_{\mathrm{A}_{\mathrm{SRS}}}=11,16$, and $21 \mu \mathrm{m}$, which yield a total number of space-resolved spectra of 142,65 , and 38 , respectively, as shown in Fig. 4. For each discretization, RADIATOR takes into account the actual lines of sight (LOS1, LOS2, and LOS3), computes all of the space-resolved spectra based on the target temperature and density distributions given by Eqs. (3) and (4).

For the object space (or implosion cores) discretization, we also tested three different values of the voxel size $\Delta \mathrm{x}_{\mathrm{voxel}}=11,16$, and $21 \mu \mathrm{m}$, and two values of threshold fraction $f_{t h}=0.5$ and 0.8. Each combination of $\Delta \mathrm{x}_{\text {voxel }}$ and $f_{\text {th }}$ provides a different number of voxels as shown in Fig. 5. As the values of $\Delta \mathrm{x}_{\text {voxel }}$ and $f_{\text {th }}$ become smaller, the spatial structure can be more flexible, but at the same time, the parameter search itself becomes more challenging due to the increase in the number of parameters and also due to the decrease in the contribution to the emergent spectra. As the threshold fraction increases, RADIATOR has to rely more on extrapolation on the edge of the implosion core. As the threshold fraction decreases, the model relies less on extrapolation, but at the same time, such a small fraction of volume on the edge may not be constrained enough by the set of space-resolved spectra.

The spatial structure of the implosion core is analyzed for each combination of discretizations on the image plane (Fig. 4) and in the object space (Fig. 5). To save computational time, we did not use the complete PGALM, but we used only the LM minimization method. For each case, three 
TABLE I. Uniqueness sensitivity to the discretizations.

\begin{tabular}{|c|c|c|c|c|c|c|c|}
\hline & & \multicolumn{6}{|c|}{ Image plane } \\
\hline & & & & $\Delta \mathrm{x}_{\mathrm{A}_{\mathrm{SE}}}$ & SRS） & & \\
\hline \multicolumn{2}{|c|}{ Object space } & \multicolumn{2}{|c|}{$11 \mu \mathrm{m}(142)$} & \multicolumn{2}{|c|}{$16 \mu \mathrm{m}(65)$} & \multicolumn{2}{|c|}{$21 \mu \mathrm{m}(38)$} \\
\hline$\Delta \mathrm{x}_{\mathrm{vxl}}$ & $f_{t h}\left(\mathrm{~N}_{\mathrm{vxl}}\right)$ & $\mathrm{U}_{\mathrm{Te}}$ & $\mathrm{U}_{\mathrm{Ne}}$ & $\mathrm{U}_{\mathrm{Te}}$ & $\mathrm{U}_{\mathrm{Ne}}$ & $\mathrm{U}_{\mathrm{Te}}$ & $\mathrm{U}_{\mathrm{Ne}}$ \\
\hline \multirow[t]{2}{*}{$21 \mu \mathrm{m}$} & $0.8(34)$ & 0.74 & 2.9 & 1.5 & 3.2 & 6.2 & 8.9 \\
\hline & $0.5(49)$ & 0.89 & 2.3 & 1.6 & 5.0 & 4.5 & 9.5 \\
\hline \multirow[t]{2}{*}{$16 \mu \mathrm{m}$} & $0.8(81)$ & 3.3 & 4.9 & 3.7 & 6.1 & 5.6 & 10. \\
\hline & $0.5(108)$ & 1.6 & 2.6 & 3.5 & 5.2 & 6.3 & 11. \\
\hline \multirow[t]{2}{*}{$11 \mu \mathrm{m}$} & $0.8(243)$ & 3.5 & 5.8 & 5.5 & 9.0 & 6.2 & 11. \\
\hline & $0.5(304)$ & 4.9 & 7.6 & 4.7 & 7.6 & 6.4 & 10. \\
\hline
\end{tabular}

different uniform conditions are, respectively, refined by the LM method to check uniqueness. The complete search starting from PGA is not necessary because the solution is known. The purpose of these runs is to find the best combination of discretizations. With a better combination of discretizations, the problem should be better constrained, and a small number of iterations of LM starting from three different uniform conditions should show good convergence and find closer spatial structure to the target.

To investigate limitations and requirements of the method, all the results have to be evaluated and compared somehow. It is useful to define a "constraint parameter" $\mathrm{P}_{\text {const }}$, which is defined as follows:

$$
\mathrm{P}_{\text {const }} \equiv \frac{\mathrm{N}_{\mathrm{SRS}}}{\mathrm{N}_{\text {voxel }}},
$$

where $\mathrm{N}_{\mathrm{SRS}}$ is the number of space-resolved spectra and $\mathrm{N}_{\text {voxel }}$ is the number of voxels. Since spatial information is provided by space-resolved spectra, the more space-resolved spectra are simultaneously and self-consistently analyzed, the more spatial information can be extracted. The decoded implosion core becomes more flexible as the number of voxels increases; but at the same time, the analysis becomes more demanding and requires more spatial information. Thus, the analysis robustness can be related to $\mathrm{P}_{\text {const }}$. We evaluated the quality of the analysis following two different criteria: the solution uniqueness and correctness. The results are summarized as a function of $\mathrm{P}_{\text {const }}$.

Starting from three different uniform initial conditions, the LM minimization found three electron temperature and density distributions. The uniqueness is quantitatively defined as

$$
\overline{\mathrm{U}}_{\mathrm{X}}=\left(\frac{1}{\mathrm{~N}_{\text {voxel }}} \sum_{\mathrm{i}=1}^{\mathrm{N}_{\text {voxel }}} \frac{\sigma_{\mathrm{X}, \mathrm{i}}}{\mu_{\mathrm{X}, \mathrm{i}}}\right) \times 100 \%
$$

where $\sigma_{\mathrm{X}, \mathrm{i}}$ and $\mu_{\mathrm{X}, \mathrm{i}}$ are the sample standard deviation and mean of the resultant variable $X$, which is either $T_{e}$ or $N_{e}$, at voxel $\mathrm{i}$ over the three results. A better combination of discretizations would constrain the problem more, which should yield smaller uniqueness parameter $\overline{\mathrm{U}}_{\mathrm{Te}}$ and $\overline{\mathrm{U}}_{\mathrm{Ne}}$. The comparisons of the solution uniqueness are summarized in Table $\mathrm{I}$, and plotted as a function of the constraint parameter $\mathrm{P}_{\text {const }}$ in Fig. 9. This figure shows an inverse relationship between the uniqueness parameter and the constraint parameter $\mathrm{P}_{\text {const }}$ (Fig. 9). The analysis results show very good solution uniqueness when $\mathrm{P}_{\text {const }}>1$, which means $\mathrm{N}_{\text {SRS }}>\mathrm{N}_{\text {voxel }}$.

The solution uniqueness is a necessary condition but not sufficient to conclude that the search is successful. The search will be successful when the found unique distribution is the correct one, which is the target distributions. Even though the parameters found by each analysis can be decoded into continuous temperature and density distributions, it is not appropriate to compare these continuous temperature and density to the target. This is because the decoded distributions contain some sharp structures created by linear interpolations, which are artificial and should not be trusted. It is more appropriate to compare $\mathrm{N}_{\text {voxel }}$ temperatures and $\mathrm{N}_{\text {voxel }}$ densities to the corresponding values of target distributions. Thus, the second criterion is the comparison of the voxel-averaged distributions between the analyses results and the target distributions. The voxel-averaged
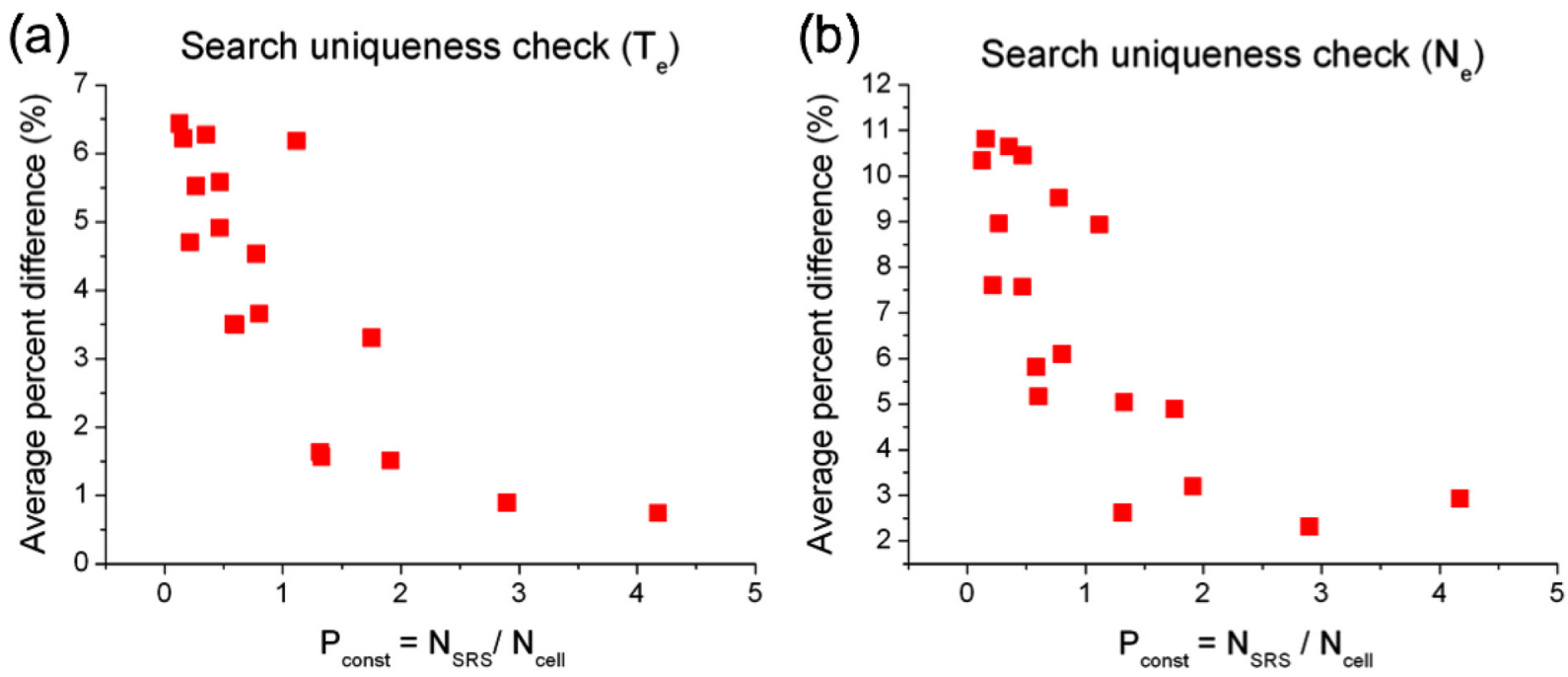

FIG. 9. Uniqueness parameter (a) $\mathrm{U}_{\mathrm{Te}}$ and (b) $\mathrm{U}_{\mathrm{Ne}}$ difference as a function of the constraint parameter $\mathrm{P}_{\text {const }}$. 
TABLE II. Analysis correctness sensitivity to the discretizations.

\begin{tabular}{|c|c|c|c|c|c|c|c|}
\hline & & \multicolumn{6}{|c|}{ Image plane } \\
\hline & & & & $\Delta \mathrm{x}_{\mathrm{A}_{\mathrm{S}}}$ & SRS） & & \\
\hline \multicolumn{2}{|c|}{ Object space } & \multicolumn{2}{|c|}{$11 \mu \mathrm{m}(142)$} & \multicolumn{2}{|c|}{$16 \mu \mathrm{m}(65)$} & \multicolumn{2}{|c|}{$21 \mu \mathrm{m}(38)$} \\
\hline$\Delta \mathrm{x}_{\mathrm{vxl}}$ & $f_{t h}\left(\mathrm{~N}_{\mathrm{vxl}}\right)$ & $\mathrm{C}_{\mathrm{Te}}$ & $\mathrm{C}_{\mathrm{Ne}}$ & $\mathrm{C}_{\mathrm{Te}}$ & $\mathrm{C}_{\mathrm{Ne}}$ & $\mathrm{C}_{\mathrm{Te}}$ & $\mathrm{C}_{\mathrm{Ne}}$ \\
\hline \multirow[t]{2}{*}{$21 \mu \mathrm{m}$} & $0.8(34)$ & 9.6 & 18. & 9.1 & 21. & 11. & 16. \\
\hline & $0.5(49)$ & 6.6 & 7.6 & 6.5 & 10. & 8.3 & 14. \\
\hline \multirow[t]{2}{*}{$16 \mu \mathrm{m}$} & $0.8(81)$ & 10. & 13. & 9.8 & 14. & 13. & 17. \\
\hline & $0.5(108)$ & 5.6 & 9.5 & 9.0 & 15. & 12. & 16. \\
\hline \multirow[t]{2}{*}{$11 \mu \mathrm{m}$} & $0.8(243)$ & 9.5 & 13. & 14. & 20. & 15. & 19. \\
\hline & $0.5(304)$ & 8.8 & 13. & 14. & 18. & 15. & 20. \\
\hline
\end{tabular}

distributions are created by computing the averages of continuous temperature and density within each voxel, which can be done for both target distributions and the continuous distributions decoded from the analysis results. Since each analysis has three solutions starting from different initial conditions, we take the average over the three solutions to come up with single voxel-averaged distribution of the analysis. To quantify the goodness of this comparison, the correctness parameter is defined as follows:

$$
\begin{gathered}
\mathrm{C}_{\mathrm{X}}=\frac{1}{\mathrm{~N}_{\text {voxel }}} \sum_{\mathrm{i}=1}^{\mathrm{N}_{\text {voxel }}} \mathrm{C}_{\mathrm{X}, \mathrm{i}}, \\
\mathrm{C}_{\mathrm{X}, \mathrm{i}}=\frac{\left|\mathrm{X}_{\mathrm{i}}^{\mathrm{target}}-\overline{\mathrm{X}}_{\mathrm{i}}^{\text {found }}\right|}{\mathrm{X}_{\mathrm{i}}^{\text {target }}} \times 100,
\end{gathered}
$$

where $X_{i}^{\text {target }}$ is the voxel-averaged value of $T_{e}$ or $N_{e}$ for voxel $\mathrm{i}$ from the target distribution, and $\overline{\mathrm{X}}_{\mathrm{i}}^{\text {found }}$ is that from an analysis result. The comparisons of these correctness parameter for different combinations of discretizations are summarized in Table II. We must note that our analysis method does not guarantee that these two voxel-averaged distributions be exactly matched, because the target continuous distribution has curvature that cannot be accurately reproduced by the limited number of parameters with tri-linear interpolation. Still, this criterion is useful to study the robustness and limitations of the analysis method as well as the solution quality dependence on the discretization. For each combination of discretizations, the correctness parameters are computed and plotted as a function of the constraint parameter, $\mathrm{P}_{\text {const }}$, as shown in Fig. 10, which shows an inverse relationship. Furthermore, the analyses with a threshold fraction of 0.5 (blue) are more accurate than those with 0.8 (red). Thus, the analysis works better with a combination of discretizations of larger $\mathrm{P}_{\text {const }}$ with threshold fraction of 0.5.

Based on these criteria discussed above, the best combination of discretizations (on the image plane and in the object space) were investigated. All the criteria favor large $\mathrm{P}_{\text {const }}$ and the smaller threshold fractions. The analysis results are very good when $\mathrm{P}_{\text {const }}$ is greater than one, which means $\mathrm{N}_{\mathrm{SRS}}>\mathrm{N}_{\text {voxel }}$. Thus, a large number of space-resolved spectra imply that more spatial information is available for the method to constrain more detailed spatial structure. A smaller value of threshold fraction means the method relies less on extrapolation and has more flexibility to accommodate the target spatial structure. There is a possibility that the analyses with the threshold fraction smaller than 0.5 produces even better results than those with $f_{t h}=0.5$. However, in terms of spectral formation, the contribution from voxels with such small fractions is not as large as the voxels that are fully inside the core boundary. Therefore, the local conditions of these small regions are not relevant, and the corresponding voxels are not included in the analysis.

One of the best cases $\left(\Delta \mathrm{x}_{A_{S \mathrm{RS}}}=11 \mu \mathrm{m}, \Delta \mathrm{x}_{\mathrm{voxel}}=21 \mu \mathrm{m}\right.$, and $\left.f_{\mathrm{t} h}=0.5\right)$ was re-analyzed with the complete PGALM. The total number of space-resolved spectra used for the analysis is 142 and the number of voxels used in the search is 49, which result in $\mathrm{P}_{\text {const }} \approx 2.9$. The complete PGALM improved the correctness parameter from $6.6 \%$ to $6.4 \%$ for the electron temperature and from $7.6 \%$ to $6.5 \%$ for the electron density. Figure 11 shows comparisons of the voxelaveraged temperature and density distributions between the
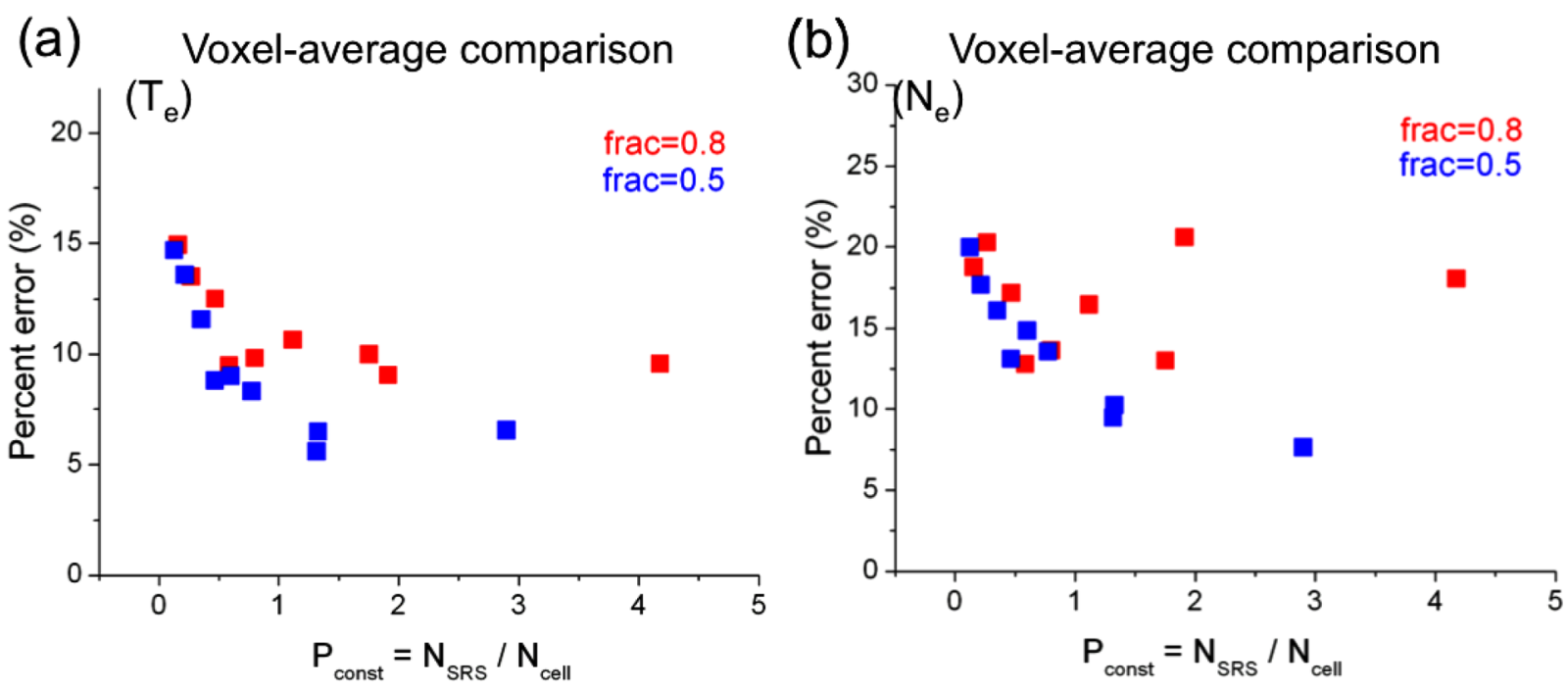

FIG. 10. Correctness parameter (a) $\mathrm{C}_{\mathrm{Te}}$ and (b) $\mathrm{C}_{\mathrm{Ne}}$ as a function of the constraint parameter $\mathrm{P}_{\text {const }}$. 


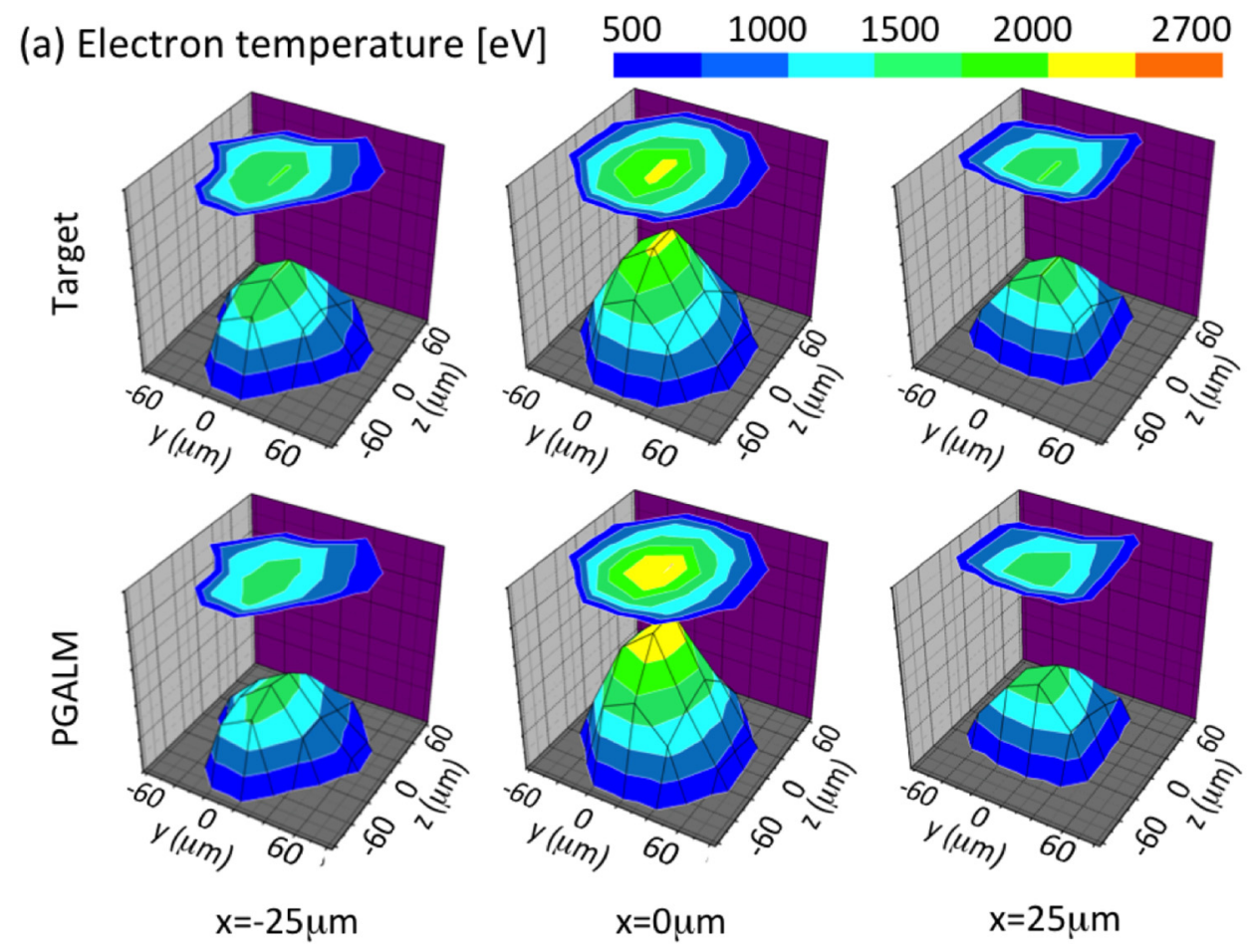

(b) Electron density $\left[10^{24} \mathrm{~cm}^{-3}\right] \quad \begin{array}{llllllll}1.0 & 1.5 & 2.0 & 2.5 & 3.0 & 3.5 & 4.0\end{array}$

FIG. 11. Comparison of the voxelaveraged distributions of (a) electron temperature and (b) density between the
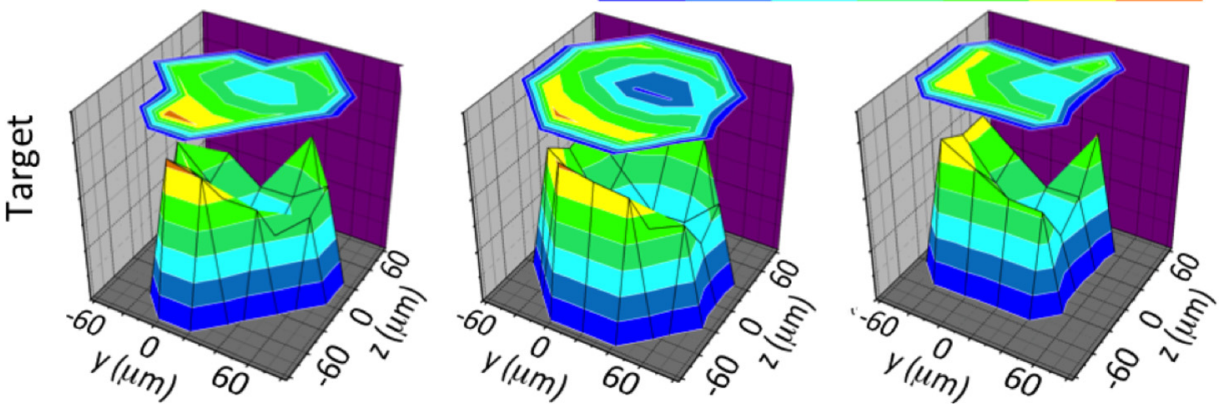

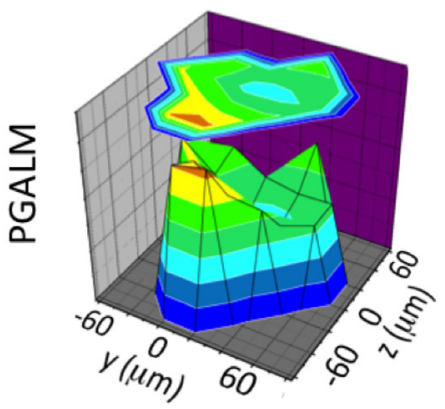

$x=-25 \mu \mathrm{m}$

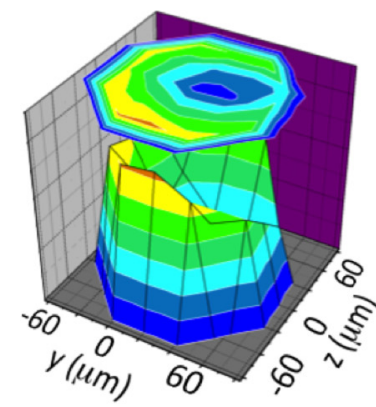

$\mathrm{x}=0 \mathrm{um}$ target and the analysis result to visually confirm how well the method works. The PGALM successfully reconstructed the spatial structure of the target implosion core including the location of the centers and the 3D features of the distributions. The largest discrepancy is found at the center of each distribution. The cause of these discrepancies could be due to either noise or discretization, or both. On one hand, as the noise on the data gets larger, the analysis results start to deviate from the true solution. On the other hand, since the decoding scheme employs tri-linear interpolation, the result-

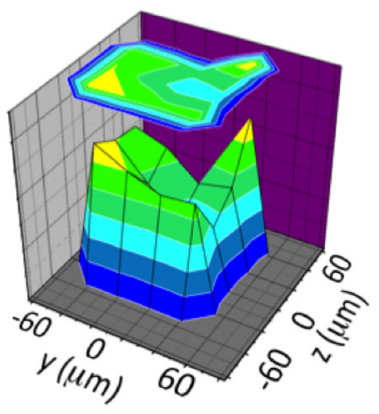

$x=25 \mu \mathrm{m}$ ant distributions will never have the same curvature that the true distribution has. Section VB will investigate if these discrepancies are created due to the noise on the data.

\section{B. Sensitivity to the noise level}

One of the critical issues in data analysis is the robustness of the method against the noise in the data. To understand how the analysis results deviate from the true solution as a function of the noise level, the two synthetic data test 


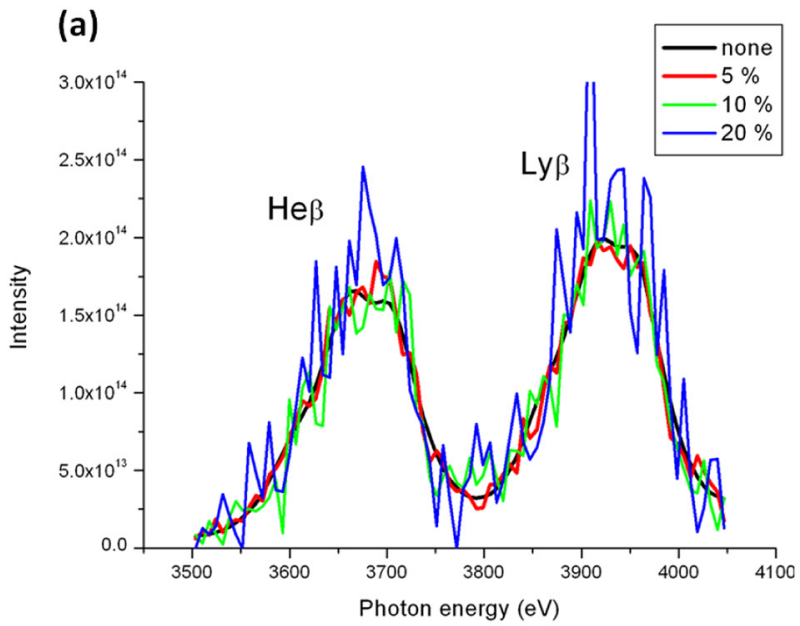

(b)

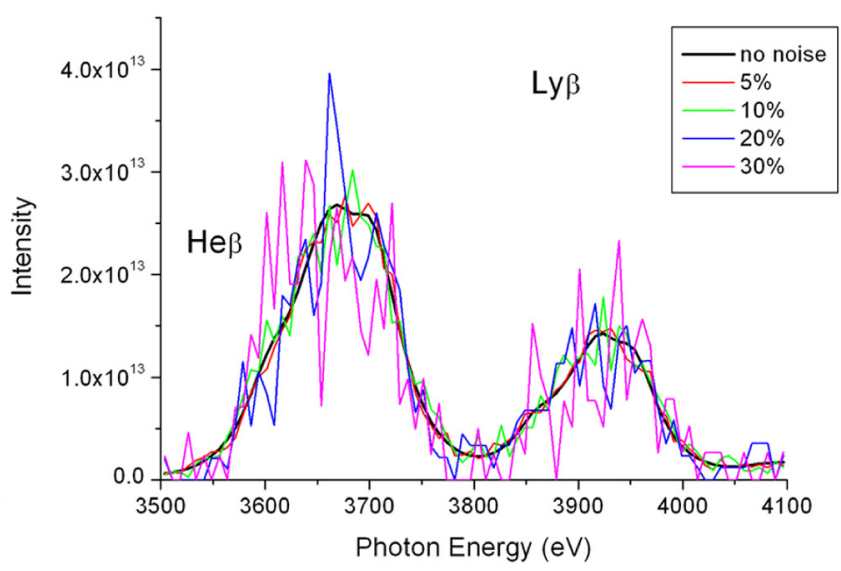

FIG. 12. Different noise levels on one of the target spectra (a) basic test case: along x-axis at (2, 2). The noise levels are $0 \%, 5 \%, 10 \%$, and $20 \%$ (b) realistic test case: along TIM4 at 26th spatial region. The noise levels are $0 \%, 5 \%, 10 \%, 20 \%$, and $30 \%$.

cases (proof-of-principle case discussed in Sec. IV and the refined test case discussed in Sec. V A) were reanalyzed using the synthetic space-resolved spectra after adding different levels of noise. The discretization parameters used for the refined test case is $\Delta \mathrm{x}_{\mathrm{voxel}}=21 \mu \mathrm{m}, f_{\text {th }}=0.5$, and $\Delta \mathrm{x}_{\mathrm{A}_{\mathrm{SRS}}}=11 \mu \mathrm{m}$. An example of how a space-resolved spectrum changes as the Poisson noise level increases is illustrated in Fig. 12(a) for proof-of-principle case, and Fig. 12(b) for the refined test case. Sets of space resolved spectra with different levels of noise are analyzed one by one (both for the proof-of-principle case and for the refined case), and the quality of each analysis is quantified by the correctness parameter defined in Eqs. (7) and (8). Then, these correctness parameters are plotted as a function of the noise level for both test cases to illustrate how analysis results deviate away from the true solution as noise level increases (Fig. 13).

There are two important points in Fig. 13. First, when there is no noise in the target spectra, the unique solution from the proof-of-principle case is identical to the target distributions, while the unique solution from the refined test case already shows small discrepancy. This implies that the discrepancy comes from the object discretization. There is no discretization problem in the proof-of-principle test case. The target temperature and densities are defined in $3 \times 3 \times 3$ voxels, and the PGALM tries to find 27 temperatures and densities in the same discretization. However, in the case of the refined test case, the target temperature and densities are continuously changing with curvature within the arbitrary volume, and the PGALM searches for a small number of temperatures and densities at centers of the voxels, so that their interpolated temperature and density distributions can best reproduce all the space-resolved spectra simultaneously. Thus, the precise curvature in the original target distribution is lost. This is why, even without any noise on the target spectra, the unique solution from the analysis yields nonzero correctness parameter.

The second important point is that, as soon as the noise level increases, the correctness parameter dramatically deteriorates for the proof-of-principle case, while it slowly deteriorates for the refined test case. This did not make sense in the fact that the proof-of-principle case is simpler in every aspect than the refined test case. However, by taking into

\section{(a) Electron temperature}

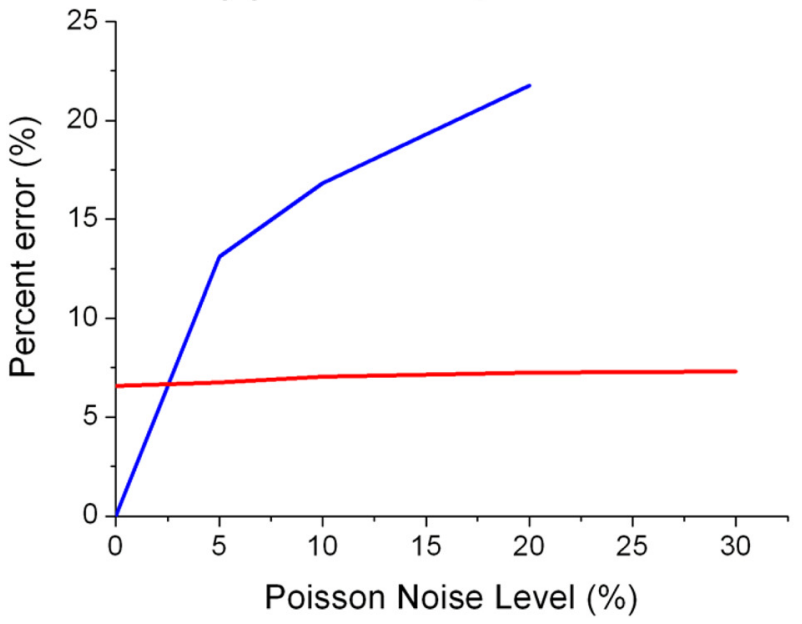

(b) Electron density

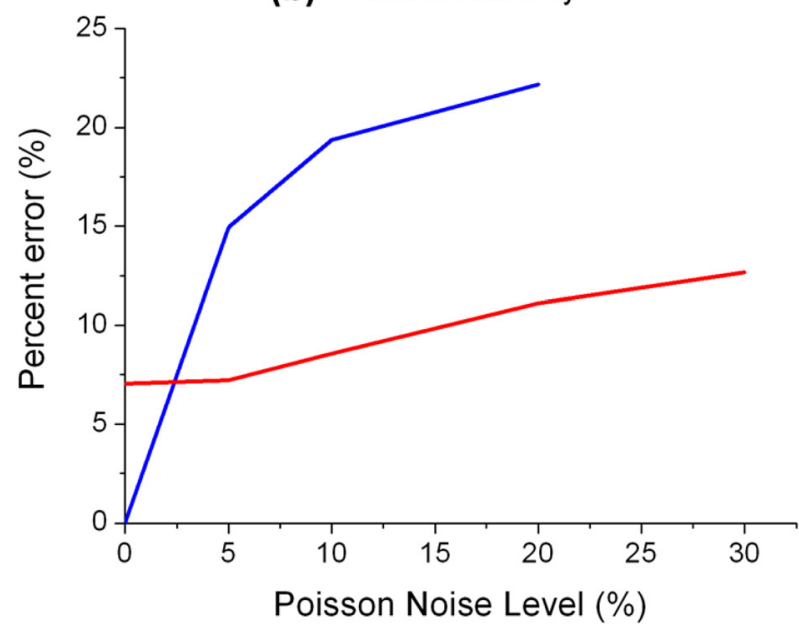

FIG. 13. Percent error as a function of noise level for basic test case (blue) and realistic test case (red) (a) electron temperature $\mathrm{T}_{\mathrm{e}}$ and (b) electron density $\mathrm{N}_{\mathrm{e}}$. 
account search requirement discussed in Sec. V A $\left(\mathrm{P}_{\text {const }}=\mathrm{N}_{\text {SRS }} / \mathrm{N}_{\text {voxel }} \geq 1\right)$, the refined test case ( $\mathrm{P}_{\text {const }}$ $=142 / 49=2.9)$ is better constrained than the proof-of-principle case $\left(\mathrm{P}_{\text {const }}=27 / 27=1\right)$. The proof-of-principle case barely satisfies the minimum constraint requirement. The given information (27 space-resolved spectra) are just enough to constrain the spatial structure (27 spatial points), and thus any noise in the target spectra would result in deviation from the true solution. The refined test case, however, has more constraints to be fit. Even if there is some noise, one spatial point contributes to many more space-resolved spectra, and to fit all of them simultaneously, the model filters some of the noise in the data, which has the same effect as smoothing or averaging. Thus, even though it does not apply any smoothing to the given spectra, the method has a sort of "self-smoothing effect" and is robust against noise when $\mathrm{P}_{\text {const }}>1$. As the constraint parameter $\mathrm{P}_{\text {const }}$ increases, the spatial structure is more constrained due to higher "selfsmoothing effect."

Figures 14 and 15 show the result of refined test case to visually confirm these points. The largest discrepancies at the centers discussed in Sec. V A are already shown in the case of no noise in the target spectra. Therefore, the discrepancies are due to the discretization and tri-linear interpolation. As the noise increases, the results for the higher noise levels are slowly deteriorating compared to those for no noise, but it still recovers the basic temperature and density distributions even with $30 \%$ Poisson noise level.

\section{SUMMARY}

We have developed a spectroscopic tool to diagnose the three-dimensional spatial structure of ICF implosion cores and investigated its limitations and requirements as well as the reliability of the results through a series of synthetic data test cases. First, geometry flexible radiation transport (RADIATOR) model was developed. RADIATOR reads in the emissivity and opacity database of the argon tracer in the implosion core, which is computed by the detailed collisional radiative model $\mathrm{ABAKO}$, and computes sets of spaceresolved spectra based on given temperature and density three-dimensional distributions defined in an arbitrary volume shape. RADIATOR is combined with a search and optimization algorithm called PGALM in order to perform the inversion, and find temperature and density distributions based on sets of space-resolved spectra extracted along lines of sight. PGALM (combined with RADIATOR) was investigated by a series of synthetic data test cases to check if it successfully extracts spatial structures and to find out its requirements and limitations.

In the proof-of-principle test case, PGALM perfectly reproduced the discrete target spatial structures (27 temperatures and densities) based on 27 space-resolved spectra computed along three orthogonal lines of sight. This test case successfully supports the idea that three-dimensional spatial structure can be constrained by sets of space-resolved spectra of along three lines of sight. Then, the method was further investigated with more realistic target spatial structure and the corresponding space-resolved spectra, which take into account additional effects such as non-orthogonality in the lines of sight, continuity in the target spatial structure, noise on the spectra, and the effect of the discretizations of the implosion core and the detector planes that have to be employed for general cases. The target is defined in the actual implosion core volume extracted from an ICF experiment, ${ }^{33}$ and temperature and density was continuously defined in the volume. The synthetic space-resolved spectra were computed along the actual three diagnostic ports in OMEGA, which are slightly off-orthogonal. Then, the method was tested under different combinations of discretizations in object space (i.e., the target implosion core) and on the image planes. The analysis results were evaluated in solution uniqueness and in solution accuracy as a function of
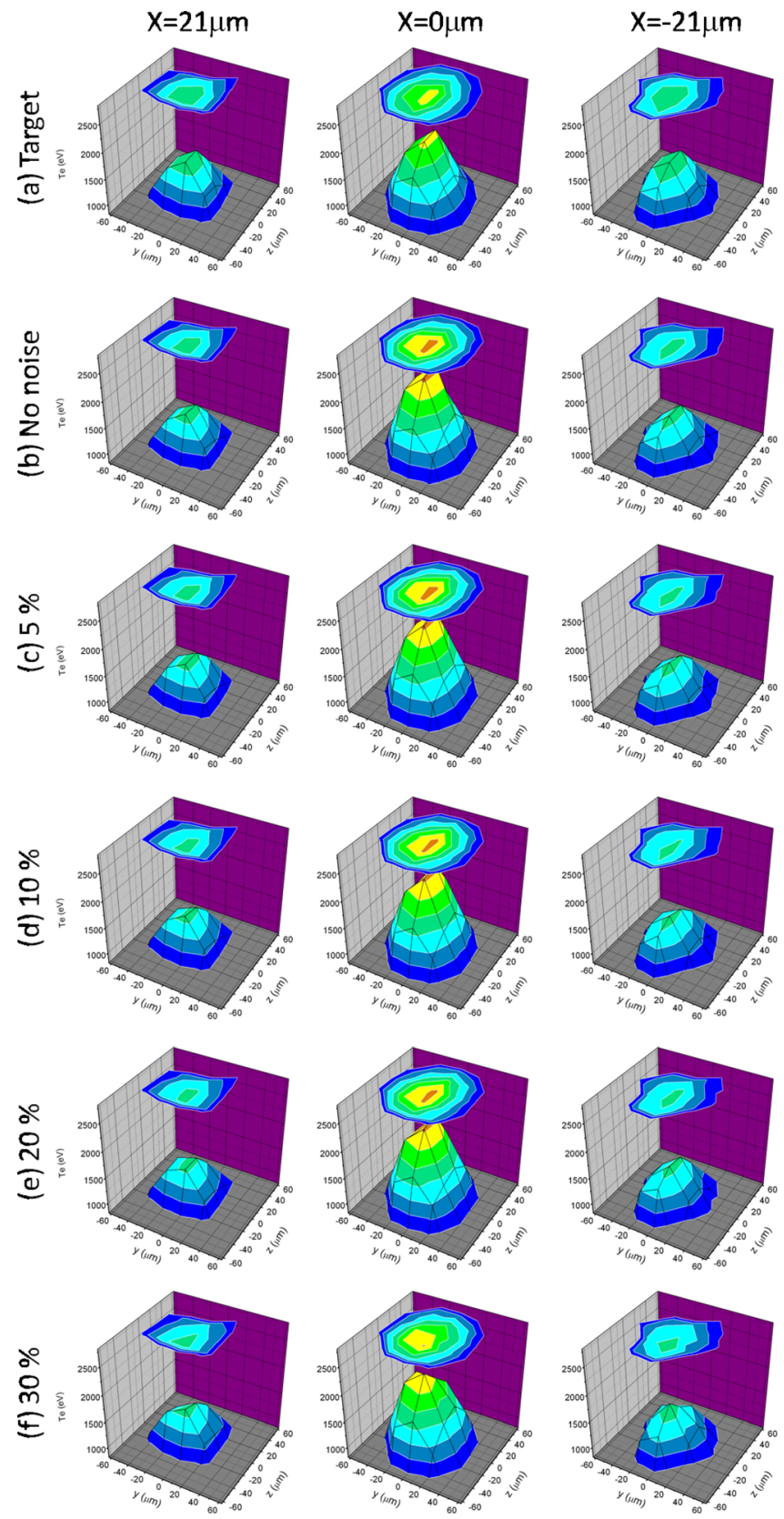

FIG. 14. Comparisons of the resultant voxel-averaged distributions of the electron temperature, based on the analysis of space-resolved spectra with different levels of noise. The results proved that the method with the analysis options employed is robust under Poisson noise. 

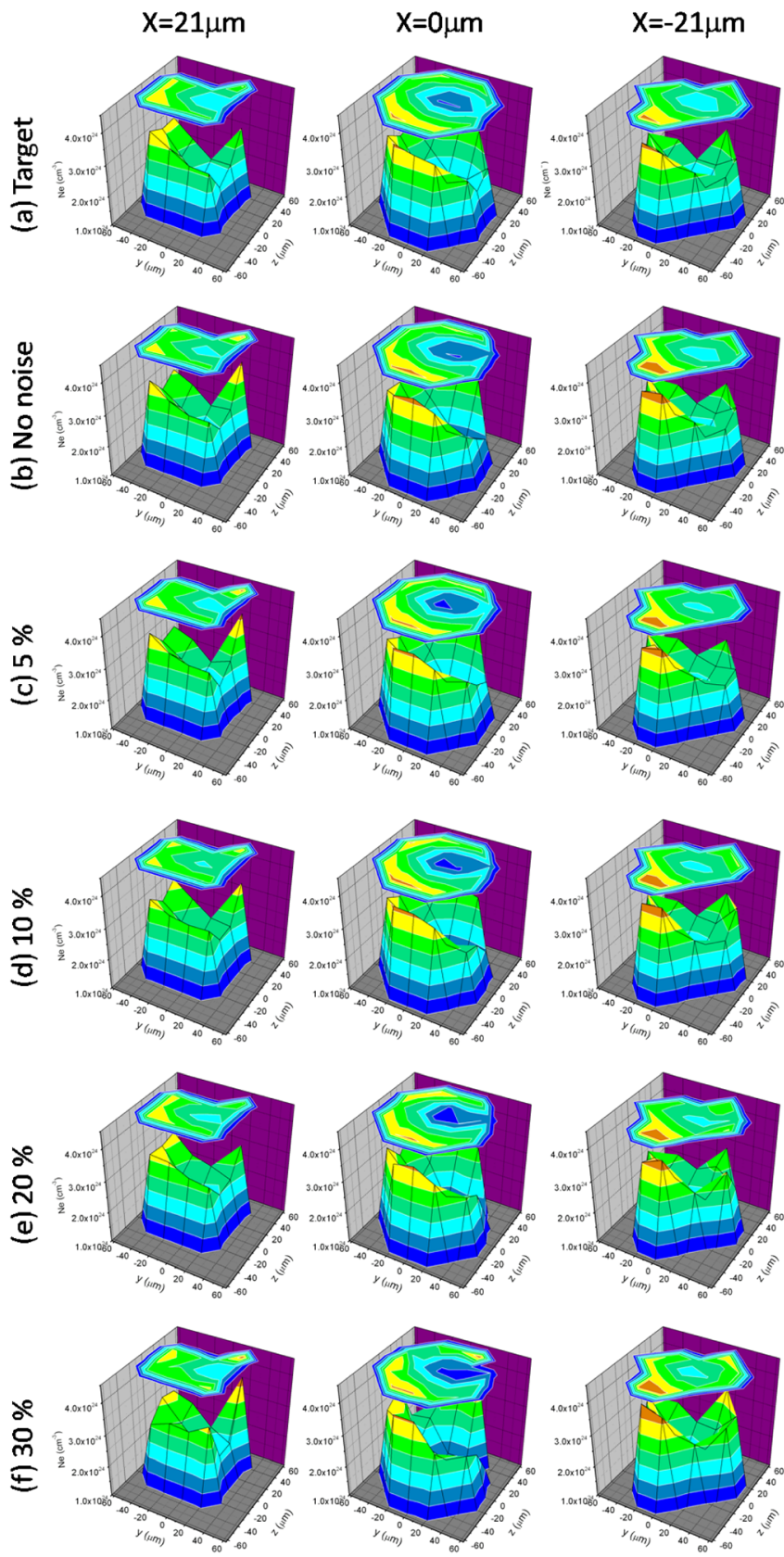

FIG. 15. Comparisons of the resultant voxel-averaged distributions of the electron density, based on the analysis of space-resolved spectra with different levels of noise. The results proved that the method with the analysis options employed is robust under Poisson noise.

constraint parameter $\mathrm{P}_{\text {const }}=\mathrm{N}_{\mathrm{SRS}} / \mathrm{N}_{\text {voxel }}$. Both the uniqueness and solution accuracy favored higher $\mathrm{P}_{\text {const }}$. When $\mathrm{P}_{\text {const }}$ is greater than 1 , the search becomes robust and the analysis results shows a few percent accuracy $(6.4 \%$ for Te and $6.5 \%$ for $\mathrm{Ne}$ ) to the target spatial structures. The noise sensitivity study indicated two interesting points of the method. One is that the analysis result shows small discrepancies even when the analyzed spectra do not contain any noise, and these discrepancies are produced by the discretization scheme and decoding scheme employed in the analysis. While the target volume has continuous distributions with arbitrary curvature, the analysis result is based on sampling points obtained with trilinear interpolation, thus the target curvature cannot be exactly reproduced, which results in discrepancies. This is a limitation of the trilinear interpolation that could be improved by using a higher-order interpolation algorithm. On the other hand, the analysis with large $\mathrm{P}_{\text {const }}$ is robust against noise in the data, and successfully recovered temperature and density spatial structures even with significant levels of noise in the data.

This work also illustrates two other points that have broader implications. First, it clearly shows the advantages of the multi-objective data analysis. In general, multiobjective data analysis extracts more information by analyzing all available data simultaneously and self-consistently. If each space-resolved spectrum were analyzed on a single and individual basis, we would extract a collection of chordaveraged temperature and density values on the image plane but not the spatial structure inside the plasma source (i.e., object space). By analyzing all the sets of space-resolved spectra simultaneously and self-consistently, PGALM successfully extracted the spatial structure of the plasma, i.e., the distribution of local values. The other advantage is its robustness against noise when the amount of data is sufficient to constrain the problem. The required $\mathrm{P}_{\text {const }}$ would probably vary depending on physical circumstances such as the optical depth of the characteristic lines, the location and the number of lines of sight used in the analysis, and so on. However, these synthetic test cases clearly illustrate that, in spite of the complexities in the search and the model, the analysis result is not very sensitive to the noise level on the data. The more pieces of data PGALM analyze simultaneously and self-consistently, the less sensitive the analysis becomes to the noise. Some information not being extracted from a piece of data due to the noise can be recovered by information given by another piece of data. Second and more importantly, this work demonstrates the feasibility of a new type of tomography, i.e., spectral or polychromatic tomography, which relies on limited LOS observations but uses information encoded in the wavelength dependence of the data.

\section{ACKNOWLEDGMENTS}

This work was supported by DOE/NLUF Grant DEFG52-09NA29042 and by Lawrence Livermore National Laboratory under Contract DE-AC52-07NA27344.

${ }^{1}$ J. Nuckolls, L. Wood, A. Thiessen, and G. Zimmerman, "Laser compresion of matter to super-high densities: Thermonuclear (ctr) applications,' Nature (London) 239, 139 (1972).

${ }^{2}$ J. Lindl, "Development of the indirect-drive approach to inertial confinement fusion and the target physics basis for ignition and gain," Phys. Plasmas 2, 3933 (1995).

${ }^{3}$ J. D. Lindl, P. Amendt, R. L. Berger, S. G. Glendinning, S. H. Glenzer, S. W. Haan, R. L. Kauffman, O. L. Landen, and L. J. Suter, "The physics basis for ignition using indirect-drive targets on the national ignition facility,' Phys. Plasmas 11, 339 (2004).

${ }^{4}$ S. Atzeni and J. Meyer-Ter-Vehn, The Physics of Inertial Fusion, edited by J. Birman, S. F. Edwards, R. Friend, M. Rees, D. Sherrington, and G. Veneziano (Clarendon, Oxford, 2004).

${ }^{5}$ H. R. Griem, "Plasma spectroscopy in inertial confinement fusion and soft X-ray laser research,” Phys. Fluids B 4, 2346 (1992).

${ }^{6}$ H. R. Griem, Principles of Plasma Spectroscopy (Cambridge University Press, 1997).

${ }^{7}$ B. A. Hammel, C. J. Keane, D. R. Kania, J. D. Kilkenny, R. W. Lee, and N. D. Delamater, "K- and 1-shell x-ray spectroscopy of indirectly driven implosions (invited), " Rev. Sci. Instrum. 63, 5017 (1992). 
${ }^{8}$ C. J. Keane, B. A. Hammel, D. R. Kania, J. D. Kilkenny, R. W. Lee, A. L. Osterheld, L. J. Suter, R. C. Mancini, J. C. F. Hooper, and N. D. Delamater, "X-ray spectroscopy of high-energy density inertial confinement fusion plasmas," Phys. Fluids B 5, 3328 (1993).

${ }^{9}$ D. A. Haynes, C. F. Hooper, Jr., R. C. Mancini, D. K. Bradley, J. Delettrez, R. Epstein, and P. A. Jaanimagi, "Spectroscopic analysis of Ardoped laser-driven implosions," Rev. Sci. Instrum. 66, 755 (1995).

${ }^{10}$ S. P. Regan, J. A. Delettrez, R. Epstein, P. A. Jaanimagi, B. Yaakobi, V. A. Smalyuk, F. J. Marshall, D. D. Meyerhofer, W. Seka, J. D. A. Haynes, I. E. Golovkin, and J. C. F. Hooper, "Characterization of direct-drive-implosion core conditions on omega with time-resolved ar k-shell spectroscopy," Phys. Plasmas 9, 1357 (2002).

${ }^{11}$ M. Tabak, J. Hammer, M. E. Glinsky, W. L. Kruer, S. C. Wilks, and R. J. Mason, "Ignition and high gain with ultrapowerful lasers," Phys. Plasmas 1, 1626 (1994).

${ }^{12} \mathrm{M}$. H. Key, "Status of and prospects for the fast ignition inertial fusion concept," Phys. Plasmas 14, 055502 (2007).

${ }^{13}$ R. Betti, C. D. Zhou, K. S. Anderson, L. J. Perkins, W. Theobald, and A. A. Solodov, "Shock ignition of thermonuclear fuel with high areal density," Phys. Rev. Lett. 98, 155001 (2007).

${ }^{14}$ W. Theobald, K. S. Anderson, R. Betti, R. S. Craxton, J. A. Delettrez, J. A. Frenje, V. Y. Glebov, O. V. Gotchev, J. H. Kelly, C. K. Li, A. J. Mackinnon, F. J. Marshall, R. L. McCrory, D. D. Meyerhofer, J. F. Myatt, P. A. Norreys, P. M. Nilson, P. K. Patel, R. D. Petrasso, P. B. Radha, C. Ren, T. C. Sangster, W. Seka, V. A. Smalyuk, A. A. Solodov, R. B. Stephens, C. Stoeckl, and B. Yaakobi, "Advanced-ignition-concept exploration on omega," Plasma Phys. Controlled Fusion 51, 124052 (2009).

${ }^{15}$ S. Atzeni, "Laser driven inertial fusion: the physical basis of current and recently proposed ignition experiments," Plasma Phys. Controlled Fusion 51, 124029 (2009).

${ }^{16}$ D. Goldberg, Genetic Algorithms in Search, Optimization, and Machine Learning (Addison-Wesley, 1989).

${ }^{17}$ I. E. Golovkin, R. C. Mancini, S. J. Louis, R. W. Lee, and L. Klein, "Analysis of X-ray spectral data with genetic algorithms," J. Quant. Spectrosc. Radiat. Transf. 75, 625 (2002).

${ }^{18}$ W. H. Press, B. P. Flannery, S. A. Teukolsky, and W. T. Vetterling, $N u$ merical Recipes in C (Cambridge University Press, 1997).

${ }^{19}$ J. J. MacFarlane, I. E. Golovkin, R. C. Mancini, L. A. Welser, J. E. Bailey, J. A. Koch, T. A. Mehlhorn, G. A. Rochau, P. Wang, and P. Woodruff, "Dopant radiative cooling effects in indirect-drive ar-doped capsule implosion experiments," Phys. Rev. E 72, 066403 (2005).

${ }^{20}$ R. Florido, T. Nagayama, R. C. Mancini, R. Tommasini, J. A. Delettrez, S. P. Regan, V. A. Smalyuk, R. Rodríguez, and J. M. Gil, "Analysis of timeresolved argon line spectra from omega direct-drive implosions," Rev. Sci. Instrum. 79, 10E310 (2008).

${ }^{21}$ R. Florido, R. C. Mancini, T. Nagayama, R. Tommasini, J. A. Delettrez, S. P. Regan, V. A. Smalyuk, R. Rodríguez, and J. M. Gil, "Argon k-shell and bound-free emssion from omega direct-drive implosion cores," High Energy Density Phys. 6, 70 (2010).

${ }^{22}$ I. Golovkin, R. Mancini, S. Louis, Y. Ochi, K. Fujita, H. Nishimura, H. Shiraga, H. Azechi, R. Butzbach, I. Uschmann, E. Forster, J. Delettrez, J. Koch, R. Lee, and L. Klein, "Spectroscopic determination of dynamic plasma gradients in implosion cores," Phys. Rev. Lett. 88, 045002 (2002).

${ }^{23}$ T. Nagayama, R. C. Mancini, L. A. Welser, S. Louis, I. E. Golovkin, R. Tommasini, J. A. Koch, N. Izumi, J. A. Delettrez, F. J. Marshall, S. P. Regan, V. A. Smalyuk, D. A. Haynes, Jr., and G. Kyrala, "Multi-objective method for fitting pinhole image intensity profiles of implosion cores driven by a pareto genetic algorithm," Rev. Sci. Instrum. 77, 10F525 (2006).

${ }^{24}$ T. Nagayama, R. C. Mancini, L. A. Welser-Sherrill, S. Louis, I. E. Golovkin, R. Tommasini, J. A. Koch, J. A. Delettrez, S. P. Regan, and V. A. Smalyuk, "Four-objective analysis including an optically thick line to extract electron temperature and density profiles in icf implosion cores," J. Phys. conf. ser. 112, 022014 (2008).

${ }^{25}$ L. A. Welser, R. C. Mancini, T. Nagayama, R. Tommasini, J. A. Koch, N. Izumi, J. A. Delettrez, F. J. Marshall, S. P. Regan, V. A. Smalyuk, I. E.
Golovkin, D. A. Haynes, Jr., and G. Kyrala, "Spatial structure analysis of direct-drive implosion cores at omega using x-ray narrow-band core images," Rev. Sci. Instrum. 77, 10E320 (2006).

${ }^{26}$ L. A. Welser, R. C. Mancini, J. A. Koch, N. Izumi, S. J. Louis, I. E. Golovkin, T. W. Barbee, Jr., S. W. Haan, J. A. Delettrez, F. J. Marshall, S. P. Regan, V. A. Smalyuk, D. A. Haynes, Jr., and R. W. Lee, "Multi-objective spectroscopic analysis of core gradients: Extension from two to three objectives," J. Quant. Spectrosc. Radiat. Transf. 99, 649 (2006).

${ }^{27}$ L. Welser-Sherrill, R. C. Mancini, J. A. Koch, N. Izumi, R. Tommasini, S. W. Haan, D. A. Haynes, I. E. Golovkin, J. J. MacFarlane, J. A. Delettrez, F. J. Marshall, S. P. Regan, V. A. Smalyuk, and G. Kyrala, "Spectroscopic determination of temperature and density spatial profiles and mix in indirect-drive implosion cores," Phys. Rev. E 76, 056403 (2007).

${ }^{28}$ T. Nagayama, R. C. Mancini, R. Florido, R. Tommasini, J. A. Koch, J. A. Delettrez, S. P. Regan, V. A. Smalyuk, L. A. Welser-Sherrill, and I. E. Golovkin, "Comparison of genetic-algorithm and emissivity-ratio analyses of image data from omega implosion cores," Rev. Sci. Instrum. 79, 10E921 (2008).

${ }^{29}$ L. A. Welser, R. C. Mancini, J. A. Koch, S. Dalhed, R. W. Lee, I. E. Golovkin, F. Marshall, J. Delettrez, and L. Klein, "Processing of multimonochromatic x-ray images from indirect drive implosions at omega," Rev. Sci. Instrum. 74, 1951 (2003).

${ }^{30}$ J. A. Koch, T. W. B. Jr., N. Izumi, R. Tommasini, R. C. Mancini, L. A. Welser, and F. J. Marshall, "Multispectral x-ray imaging with a pinhole array and a flat bragg mirror," Rev. Sci. Instrum. 76, 073708 (2005).

${ }^{31}$ N. Izumi, T. W. Barbee, J. A. Koch, R. C. Mancini, and L. A. Welser, "Reconstruction of quasimonochromatic images for multispectral $\mathrm{x}$-ray imaging with a pinhole array and a flat bragg mirror," Rev. Sci. Instrum. 77, 083504 (2006).

${ }^{32}$ R. Tommasini, J. A. Koch, N. Izumi, L. A. Welser, R. C. Mancini, J. Delettrez, S. P. Regan, and V. Smalyuk, "Narrow-band x-ray imaging for core temperature and density maps retrieval of direct drive implosions," SPIE 6317, 31716 (2006).

${ }^{33}$ T. Nagayama, R. C. Mancini, R. Florido, R. Tommasini, J. A. Koch, J. A. Delettrez, S. P. Regan, and V. A. Smalyuk, "Processing of spectrally resolved x-ray images of inertial confinment fusion implosion cores recorded with multimonochromatic x-ray imagers," J. Appl. Phys. 109, 093303 (2011).

${ }^{34}$ R. Tommasini, J. A. Koch, N. Izumi, L. A. Welser, R. C. Mancini, J. Delettrez, S. Regan, and V. Smalyuk, "Multispectral x-ray imaging for core temperature and density maps retrieval in direct drive implosions," Rev. Sci. Instrum. 77, 10E303 (2006).

${ }^{35}$ R. Florido, R. Rodríguez, J. M. Gil, J. G. Rubiano, P. Martel, E. Mínguez, and R. C. Mancini, "Modeling of population kinetics of plasmas that are not in local thermodynamic equilibrium, using a versatile collisionalradiative model based on analytical rates," Phys. Rev. E 80, 056402 (2009).

${ }^{36}$ M. F. Gu, "The flexible atomic code," Can. J. Phys. 86, 675 (2008).

${ }^{37}$ J. Bauche, C. Bauche-Arnoult, and M. Klapisch, "Transition arrays in the spectra of ionized atoms," Adv. At. Mol. Phys. 23, 131 (1987).

${ }^{38}$ J. C. Stewart and J. K. D. Pyatt, "Lowering of ionization potentails in plasmas," Astrophys. J. 144, 1203 (1966).

${ }^{39}$ R. C. Mancini, R. F. Joyce, and J. C. F. Hooper, "Escape factors for starkbroadened line profiles," J. Phys. B 20, 2975 (1987).

${ }^{40}$ C. A. Iglesias, H. E. DeWitt, J. L. Lebowitz, D. MacGowan, and W. B. Hubbard, "Low-frequency electric microfield distributions in plasmas," Phys. Rev. A 31, 1698 (1985).

${ }^{41}$ L. A. Woltz and J. C. F. Hooper, "Calculation of spectral line profiles of multielectron emitters in plasmas," Phys. Rev. A 38, 4766 (1988).

${ }^{42}$ R. C. Mancini, D. P. Kilcrease, L. A. Woltz, and J. C. F. Hooper, "Calculational aspects of the stark line broadening of multielectron ions in plasmas," Comput. Phys. Commun. 63, 314 (1991).

${ }^{43}$ D. A. Haynes, D. T. Garber, C. F. Hooper, Jr., R. C. Mancini, Y. T. Lee, D. K. Bradley, J. Delettrez, R. Epstein, and P. A. Jaanimagi, "Effects of ion dynamics and opacity on stark-broadened argon line profiles," Phys. Rev. E 53, 1042 (1996). 